\title{
Cool core remnants in galaxy clusters ${ }^{\star}$
}

\author{
M. Rossetti and S. Molendi \\ Istituto di Astrofisica Spaziale e Fisica Cosmica, INAF, via Bassini 15, 20133 Milano, Italy \\ e-mail: rossetti@iasf-milano.inaf.it
}

Received 20 August 2009 / Accepted 20 October 2009

\section{ABSTRACT}

\begin{abstract}
Context. X ray clusters are conventionally divided into two classes: "cool core" (CC) and "non-cool core" (NCC) objects, on the basis of the observational properties of their central regions. Recent results have shown that the cluster population is bimodal.

Aims. We want to understand whether the observed distribution of clusters is due to a primordial division into two distinct classes or rather to differences in the way these systems evolve across cosmic time.

Methods. We systematically search the ICM of NCC clusters in a subsample of the B55 flux limited sample of clusters for regions which have some characteristics typical of cool cores, namely low entropy gas and high metal abundance.

Results. We find that most NCC clusters in our sample host regions reminiscent of CC, i.e. characterized by relative low entropy gas (albeit not as low as in CC systems) and a metal abundance excess. We have dubbed these structures "cool core remnants", since we interpret them as the remains of a cool core after a heating event (AGN giant outbursts in a few cases and more commonly mergers). We infer that most NCC clusters have undergone a cool core phase during their life. The fact that most cool core remnants are found in dynamically active objects provides strong support to scenarios where cluster core properties are not fixed "ab initio" but evolve across cosmic time.
\end{abstract}

Key words. galaxies: clusters: general - X-rays: galaxies: clusters

\section{Introduction}

Galaxy clusters are often divided by X-ray astronomers into two classes: "cool core"(CC) and "non-cool core" (NCC) clusters. The former are characterized by a set of properties: a prominent surface brightness peak, usually roughly coincident with the center of the large scale X-ray isophotes and with the position of the brightest central galaxy (BCG), associated with a decrease of the temperature profile in the inner regions and a positive gradient of the metal abundance profile. In the central regions of these clusters, the cooling time is significantly smaller than the Hubble time, but the radiative losses are balanced by some form of heating, which in the currently prevailing scenario is attributed to the central AGN, which prevents the gas from cooling indefinitely and flowing inward. NCC clusters are characterized by the lack of these observational features. Several indicators based on these observational characteristics have been proposed to classify clusters into these categories: e.g. temperature drop (Sanderson et al. 2006, 2009b), cooling time (Bauer et al. 2005), a composition of these two criteria (Dunn et al. 2005; Dunn \& Fabian 2008), the slope of the gas density profile at a given radius (Vikhlinin et al. 2007) and core entropy (Cavagnolo et al. 2009). In a recent paper (Leccardi et al. 2010, hereafter Paper I), we suggested a robust indicator to classify clusters (see also Sect. 3), based on pseudo-entropy gradients. The classification based on this indicator improves the traditional classification scheme based on the temperature drop and is essentially equivalent to classifications based on the cooling time. Increasing attention has been devoted to the statistics of $\mathrm{CC}$, both in the local Universe and at higher redshifts. While the precise results depend strongly on the indicator used to classify clusters, the fraction of $\mathrm{CC}$ is considered to be about $50 \%$ of

^ Appendices are only available in electronic form at http: //www . aanda. org the clusters population. There are some indication that the cluster population is bimodal (Cavagnolo et al. 2009), but there are also some intermediate objects which are not easily classified (Paper I).

One of the open questions in the study of galaxy clusters concerns the origin of this distribution. The original model which prevailed for a long time assumed that the CC state was a sort of "natural state" for the clusters, and the observational features were explained within the context of the old "cooling flow" model: radiation losses cause the gas in the centers of these clusters to cool and to flow inward. Clusters were supposed to live in this state until disturbed by a "merger". Indeed, mergers are very energetic events that can shock-heat (Burns et al. 1997) and mix the ICM (Gómez et al. 2002; Ritchie \& Thomas 2002): through these processes they were supposed to efficiently destroy cooling flows. After the mergers, clusters were supposed to relax and go back to the cooling flow state in a sort of cyclical evolution. With the fall of the "cooling flow" brought about by the XMM-Newton and Chandra observations (e.g. Peterson et al. 2001; Molendi \& Pizzolato 2001), doubts were cast also on the interpretation of mergers as the dominant mechanism which could transform CC clusters into NCC. More generally speaking, the question arose whether the observed distribution of clusters was due to a primordial division into the two classes or rather to evolutionary differences during the history of the clusters.

McCarthy et al. (2004, 2008) noticed the absence of systems that resemble observed NCC clusters in cosmological simulations, suggesting that mergers could not be the origin of the cluster distribution. They proposed that early episodes of nongravitational pre-heating may explain the dichotomy; they envisage a scenario in which NCC clusters have been pre-heated to levels greater than $\sim 300 \mathrm{keV} \mathrm{cm}^{2}$ and did not have enough time to develop a cool core, while CC clusters have been pre-heated to lower levels and need an additional source of present-day 
heating to offset cooling. With this model, McCarthy et al. (2008) explained observed entropy and gas density Chandra profiles for CC and ROSAT gas density profiles for NCC. O'Hara et al. (2006) supported the primordial model, showing that the scatter in scaling relations is larger for CC clusters than for NCC, suggesting that $\mathrm{CC}$ are not more relaxed than NCC systems. Moreover the two-body idealized simulations by Poole et al. (2008) showed that mergers cannot produce extended "warm" cores and cannot destroy metal abundance gradients.

However, the evolutionary "merger" scenario has been continuously supported by observations. For instance, Sanderson et al. (2006) compared temperature and cooling time profiles of a sample of clusters observed by Chandra with indicators of merger activity and suggested that mergers may be the primary factor in preventing the formation of CCs. More recently, Sanderson et al. (2009a) have shown in a large sample of objects that the X-ray/BCG projected offset correlates with the gas density profile, which can be considered an indicator of the CC state. If the X-ray/BCG offset "measures" the dynamical state of the cluster, this result implies that the cool core strengths diminishes in more dynamically disturbed clusters. Another indicator of dynamical activity is the presence of extended radio halos on Mpc scales, whose origin is likely related to turbulent acceleration driven by mergers (Ferrari et al. 2008, for a recent review). None of the clusters which are found to host a radio halo (on Mpc scales) in a complete survey (Venturi et al. 2008; Brunetti et al. 2009) can be classified as a CC object. These relations are indeed expected if mergers can efficiently destroy cool cores.

An interesting model has been proposed by Motl et al. (2004), who suggested a double role for mergers in the cluster formation process: while they can shock-heat the ICM, they also efficiently mix the ICM and participate in the formation of $\mathrm{CCs}$ by providing cool gas. By analyzing simulated temperature and surface brightness maps, they find that observational signatures of a cool core may disappear after mergers, but that cool gas remains in the systems at all time. Indeed, mergers are complicated phenomena during which the ICM of the interacting objects is mixed. Also the simulations by Ascasibar \& Markevitch (2006) on the formation of cold fronts showed that an interacting subcluster may donate its cool gas to the main cluster and that, during the merging processes, many hydrodynamic effects contribute to the mixing of the gas.

Recently, we found an unexpected and interesting result from the analysis of the metalicity profiles in a large sample of clusters (Paper I): some clusters that cannot be classified as CC show a metal abundance excess at their center, without a significant temperature decline or an X-ray brightness excess. We suggested that at least some NCC clusters have spent part of their lives as $\mathrm{CC}$ objects and that consequently a complete primordial separation of the two classes of objects cannot be the case.

However, further analysis is needed on this result to use it to gain insight on the origin of the distribution of CC-NCC objects. We would like to characterize these regions better and to know if and to what extent they are common in the population of NCC objects. The results in Paper I as well as those described above comparing observations and simulation, are based on "uni-dimensional" properties (entropy and metal abundance profiles). Indeed, global properties and unidimensional profiles are easy to derive and it is possible to compare them quantitatively, but they inevitably result in a loss of information. Many thermodynamic maps are now available in the literature, which show that spherical symmetry is not generally fulfilled in clusters, and which reveal the presence of regions with "anomalous" characteristics outside of the cores (e.g. Sun et al. 2002; Rossetti et al. 2007, and many others).

In this paper, we present a systematic two-dimensional analysis of a sample of 35 clusters observed with XMM-Newton, to look for and characterize regions with a significant metal excess in NCC clusters, like the ones found in Paper I. The outline of the paper is as follows: in Sect. 2 we describe the sample and the data analysis, in Sect. 3 we discuss the classification schemes and we define "CC remnants" in Sect. 4. We interpret our results in Sect. 5, and we summarize our findings in Sect. 6. Quoted confidence intervals are $68 \%$ for one interesting parameter unless otherwise stated. All results are given assuming a $\Lambda$ CDM cosmology with $\Omega_{\mathrm{m}}=0.3, \Omega_{\Lambda}=0.7$, and $H_{0}=70 \mathrm{~km} \mathrm{~s}^{-1} \mathrm{Mpc}^{-1}$.

\section{Data analysis}

\subsection{The sample}

The starting point of the sample of galaxy clusters described in this paper is the "B55" X-ray flux limited sample (Edge et al. 1990). In order to have a significant coverage of the cluster within EPIC field of view, we have eliminated the nearest clusters and considered only those with redshift $z>0.03$. Then we analyzed all public XMM-Newton observations, including mosaics and multiple observations, as described in Sect. 2.2. We discarded observations where, after soft proton cleaning, the effective exposure time (MOS1 1 MOS2 + pn) is lower than $25 \mathrm{ks}$, except in the case of mosaics where observations with $t_{\exp }<25 \mathrm{ks}$ have been used for images but not for spectral analysis.

The list of the clusters which remained after our selection criteria is given in Table 1. A644, A2244 have not been observed by XMM-Newton, while all the observations of A3391, A1736, A2142, A2063 and A1651 are badly contaminated by soft protons $\left(t_{\exp }<25 \mathrm{ks}\right)$ and have been discarded. The cluster Cygnus $\mathrm{A}$ has been discarded in a second phase of the analysis because of the presence of the radio galaxy QSO B1957+405, featuring large hotspots well detected in X-rays, which cannot be easily subtracted from the core spectrum.

\subsection{General data reduction}

We retrieve Observation Data Files (ODF) from the XMM-Newton archive and process them with SAS software version 7.0. The event files produced by this standard analysis technique are then cleaned to remove soft proton flares with a double filtering process. As a first step, we produce the light curve in a hard energy band (10-12 keV) and remove all time periods with count rates exceeding a fixed threshold $0.025 \mathrm{cts} / \mathrm{s}$ for the MOS detectors and $0.050 \mathrm{cts} / \mathrm{s}$ for the pn. This procedure allows the removal of most flares, but softer flares may survive. In the second step, we apply a $\sigma$ clipping technique to the histogram obtained from the light curve in the 2-5 keV energy range. For each observation, we have calculated the "in over out ratio", $R_{\mathrm{SB}}$ (De Luca \& Molendi 2004), to identify observations badly contaminated by a quiescent soft proton component. As outlined in Paper I, the ratio has been calculated in an external annulus at $E>9 \mathrm{keV}$ to reduce the contribution of the cluster emission which fills all the FOV, especially for the nearest objects. We have discarded only one observation (and therefore the cluster A2065) where the $R_{\mathrm{SB}}$ of the two MOS is larger than 2.0. After soft proton cleaning we filter the event files according to pattern and flag criteria, and we remove by sight brightest point sources. 
Since we are mainly interested in characterizing the thermodynamic properties of the ICM in the central and brightest regions of the clusters of our sample, advanced procedures to treat the background (Leccardi \& Molendi 2008b) are not strictly necessary. This is also the reason why we could discard only badly contaminated observations with $R_{\mathrm{SB}}>2.0$. As background event files, we merge nine "blank sky" field observations, as is commonly done, and we extract images and spectra for the background in the same way than for the source observations. We calculate a normalization factor $Q$ for each cluster observation to take into account possible temporal variations of the instrumental background. The normalization factor is the count rate ratio between source and background observations in an external ring $\left(10^{\prime}-12^{\prime}\right)$ beyond $9 \mathrm{keV}$. Background images and spectra are scaled by $Q$ before the subtraction from source images and spectra.

\subsection{Two-dimensional analysis}

For all the clusters in our sample we prepared two dimensional maps of the main thermodynamic quantities, starting from EPIC images. To do this we have used a modified version of the adaptive binning + broad band fitting technique described in Rossetti et al. (2007), where we have substituted the Cappellari \& Copin (2003) adaptive binning algorithm with the weighted Voronoi tessellation by Diehl \& Statler (2006) (Rossetti 2006).

Especially in clusters undergoing major mergers, where there is no spherical symmetry, maps are a fundamental tool to select interesting regions for a proper spectral analysis, which is necessary to complement the thermodynamical information with the chemical information. Indeed, a "blind" spectral analysis in concentric annuli would not allow to detect interesting features in some of our clusters.

\subsection{One-dimensional analysis}

\subsubsection{Profiles}

As a first step, we have performed spectral extraction and analysis in concentric annuli. As discussed in Sect. 2.3, the main drawback of this technique is that the assumption of spherical symmetry is not always fulfilled in our clusters, and in some cases even the choice of the center can have a strong impact on the observed properties. Therefore we have used the thermodynamic maps (Sect. 2.3) to identify those clusters where the deviations from the spherical symmetry are larger, and we have decided not to perform radial analysis in four well known merging objects: A3667 (Briel et al. 2004), A2256 (Sun et al. 2002), A754 (Henry et al. 2004) and A3266 (Finoguenov et al. 2006).

For the remaining clusters of the sample, where we do not observe large displacements between the surface brightness peak and the entropy minimum, we select as center of symmetry the surface brightness peak, even if the large scale isophotes have another center. This allows a better description of the ICM properties in the more central regions of the clusters.

We extract spectra from annular regions around the selected center. For each instrument (MOS1, MOS2 and pn) and each region we extract source and background spectra, and we generate an effective area (ARF) file. Then we associate a redistribution matrix file to the spectrum, appropriated for the instrument and, in the pn case, for the position of the selected region in the detector.

We perform spectral fitting, using the XSPEC v11.3 package, separately for each spectrum in the energy range $0.5-10 \mathrm{keV}$.
We use an absorbed mekal model (WABS*MEKAL), where the $N_{\mathrm{H}}$ is fixed to the input galactic value ${ }^{1}$ and the redshift is allowed to vary in a small range (width $\simeq 0.02$ ) around the nominal value. Temperature, metal abundance ${ }^{2}$ and normalization are the free parameters of the fit (following the prescription in Leccardi \& Molendi 2008a, even negative values are allowed for the metal abundance). Best fit results obtained from the three instruments and from multiple observations of the same region are then averaged together with a weighted mean. This enables us to produce the projected temperature, metal abundance and surface brightness profiles for each cluster.

Finally, we perform a deprojection of the profiles, following the technique described in Ettori et al. (2002), to derive the threedimensional density and temperature profiles. Combining them, we obtain entropy and cooling time profiles.

\subsubsection{IN and OUT regions}

In order to classify clusters according to the scheme described in Paper I, we extracted spectra in an IN region and an OUT reference region, whose radii are defined as a fixed fraction of $R_{180}$ ( $r<0.05 R_{180}$ and $0.05 R_{180}<r<0.2 R_{180}$, respectively). $R_{180}$ has been calculated as

$R_{180}=1780\left(\frac{k T}{5 \mathrm{keV}}\right)^{1 / 2} h(z)^{-1} \mathrm{kpc}$,

where $h(z)=\left(\Omega_{m}(1+z)^{3}+\Omega_{\Lambda}\right)^{1 / 2}$ and $k T$ is the temperature of the cluster (Arnaud et al. 2005; Leccardi \& Molendi 2008b). This mean temperature has been first calculated starting from the profiles (eventually excluding the bins of the temperature drop in CC clusters). After the extraction of the OUT spectra, we have re-calculated $R_{180}$ using as mean temperature $T_{\text {out }}$, i.e. the temperature obtained with a spectral fit in the region $0.05 R_{180}<r<0.2 R_{180}$. We have then calculated the differences between the new estimate of $0.2 R_{180}$ and the old one. Once converted in arcseconds, we have compared this difference with the XMM-Newton point spread function: if the two estimates of $0.2 R_{180}$ differed by more than $15^{\prime \prime}$, we extracted again spectra with the new $R_{180}$ and iteratively repeated the procedure until the differences were smaller than the PSF. In the case of the four clusters without radial profiles (A754, A2256, A3266 and A3667), the first estimate of the mean temperatures has been performed directly from the temperature maps. The list of the final $T_{\text {out }}$ and $R_{180}$ can be found in Table 2 .

As discussed in Sect. 2.3, the choice of the center has been performed starting from the thermodynamic maps. More specifically, we have selected as a center the position of the minimum in the pseudo-entropy map. In most cases, the position of the entropy minimum coincides with the surface brightness peak, while in other clusters there is a significant displacement, but usually smaller then the radius of the IN regions, with the exception of A3667. Moreover the choice of the position of the entropy minimum as a center instead of the surface brightness peak does not significantly alter the value of the pseudo entropy ratio, except for the case of A3667. This is a well studied merging cluster (Vikhlinin et al. 2001; Briel et al. 2004): the low-entropy gas is concentrated at the position of a prominent cold front about $500 \mathrm{kpc}$ SE from the surface brightness peak (see the figure in Appendix B).

\footnotetext{
1 The only exception is A478, where we found a difference of about a factor of two between the galactic value $\left(1.48 \times 10^{21} \mathrm{~cm}^{2}\right.$, Dickey \& Lockman 1990) and the best fit value $\left(2.7 \times 10^{21} \mathrm{~cm}^{2}\right)$.

${ }^{2}$ For solar abundances we refer to Anders \& Grevesse (1989).
} 
A\&A 510, A83 (2010)

Table 2. Properties of the clusters in the sample: the first 14 entries are LEC clusters, while the remaining are non-LEC.

\begin{tabular}{|c|c|c|c|c|c|c|}
\hline Name & Redshift & $\begin{array}{c}\text { Temperature } \\
(\mathrm{keV})\end{array}$ & $\begin{array}{c}R_{180} \\
(\mathrm{Mpc})\end{array}$ & $\sigma$ & Core $\mathrm{Cl}$. & Dyn. Cl. \\
\hline A2199 & 0.0301 & $4.101 \pm 0.025$ & 1.58 & $0.4216 \pm 0.0033$ & $\mathrm{CC}$ & NOM \\
\hline A496 & 0.0329 & $4.990 \pm 0.038$ & 1.75 & $0.3109 \pm 0.0028$ & $\mathrm{CC}$ & $\mathrm{NOM}^{*}$ \\
\hline $2 \mathrm{~A} 0335+096$ & 0.035 & $3.649 \pm 0.009$ & 1.49 & $0.2644 \pm 0.0007$ & $\mathrm{CC}$ & NOM \\
\hline A2052 & 0.035 & $2.884 \pm 0.014$ & 1.33 & $0.4274 \pm 0.0028$ & $\mathrm{CC}$ & NOM \\
\hline A4059 & 0.047 & $3.968 \pm 0.028$ & 1.55 & $0.4394 \pm 0.0044$ & $\mathrm{CC}$ & NOM \\
\hline Hydra A & 0.054 & $3.413 \pm 0.024$ & 1.43 & $0.4138 \pm 0.0040$ & $\mathrm{CC}$ & NOM \\
\hline A85 & 0.055 & $5.491 \pm 0.048$ & 1.82 & $0.3857 \pm 0.0050$ & $\mathrm{CC}$ & MRG \\
\hline A1795 & 0.062 & $5.416 \pm 0.026$ & 1.80 & $0.3524 \pm 0.0023$ & $\mathrm{CC}$ & $\mathrm{NOM}$ \\
\hline A3112 & 0.075 & $4.319 \pm 0.033$ & 1.59 & $0.3855 \pm 0.0038$ & $\mathrm{CC}$ & NOM \\
\hline A2597 & 0.085 & $3.491 \pm 0.018$ & 1.43 & $0.3568 \pm 0.0026$ & $\mathrm{CC}$ & NOM \\
\hline A478 & 0.088 & $7.057 \pm 0.034$ & 2.03 & $0.3706 \pm 0.0024$ & $\mathrm{CC}$ & NOM $^{*}$ \\
\hline PKS0745-191 & 0.103 & $7.765 \pm 0.12$ & 2.11 & $0.2957 \pm 0.0056$ & $\mathrm{CC}$ & $\mathrm{NOM}^{*}$ \\
\hline A2204 & 0.152 & $7.412 \pm 0.092$ & 2.01 & $0.2972 \pm 0.0046$ & $\mathrm{CC}$ & NOM \\
\hline A2029 & 0.077 & $6.173 \pm 0.056$ & 1.90 & $0.4505 \pm 0.0057$ & $\mathrm{CC}$ & NOM \\
\hline A4038 & 0.030 & $2.957 \pm 0.015$ & 1.35 & $0.5093 \pm 0.0037$ & INT & NOM \\
\hline A576 & 0.039 & $3.777 \pm 0.044$ & 1.52 & $0.6226 \pm 0.0135$ & INT & MRG \\
\hline A3571 & 0.039 & $6.253 \pm 0.045$ & 1.95 & $0.6220 \pm 0.0078$ & INT & NOM \\
\hline A119 & 0.044 & $6.032 \pm 0.082$ & 1.92 & $0.7621 \pm 0.0279$ & NCC & MRG \\
\hline MKW3s & 0.045 & $3.325 \pm 0.018$ & 1.42 & $0.4860 \pm 0.0039$ & $\mathrm{CC}$ & NOM \\
\hline A1644 & 0.047 & $4.156 \pm 0.056$ & 1.59 & $0.5318 \pm 0.0132$ & INT & NOM \\
\hline A3558 & 0.048 & $5.221 \pm 0.025$ & 1.78 & $0.6155 \pm 0.0057$ & INT & NOM \\
\hline A3562 & 0.048 & $4.344 \pm 0.032$ & 1.62 & $0.6089 \pm 0.0083$ & $\mathrm{NCC}$ & MRG \\
\hline Triangulum Australis & 0.051 & $9.224 \pm 0.095$ & 2.36 & $0.6845 \pm 0.0164$ & $\mathrm{NCC}$ & NOM \\
\hline A 3158 & 0.060 & $4.903 \pm 0.042$ & 1.71 & $0.7488 \pm 0.0153$ & NCC & MRG \\
\hline A399 & 0.072 & $5.984 \pm 0.109$ & 1.88 & $0.7086 \pm 0.0319$ & $\mathrm{NCC}$ & MRG \\
\hline A401 & 0.074 & $7.268 \pm 0.084$ & 2.07 & $0.7028 \pm 0.0212$ & NCC & MRG \\
\hline A2255 & 0.081 & $6.197 \pm 0.154$ & 1.91 & $0.8853 \pm 0.0714$ & $\mathrm{NCC}$ & MRG \\
\hline A1650 & 0.084 & $5.427 \pm 0.0539$ & 1.78 & $0.5323 \pm 0.0089$ & INT & NOM \\
\hline A1689 & 0.183 & $8.612 \pm 0.102$ & 2.13 & $0.4724 \pm 0.0089$ & INT & NOM \\
\hline A2319 & 0.056 & $8.962 \pm 0.091$ & 2.32 & $0.5643 \pm 0.0113$ & NCC & MRG \\
\hline A3532 & 0.055 & $4.839 \pm 0.104$ & 1.70 & $0.7285 \pm 0.0336$ & INT & $\mathrm{NOM}^{*}$ \\
\hline A3667 & 0.056 & $5.461 \pm 0.029$ & 1.81 & $0.6378 \pm 0.0078$ & $\mathrm{NCC}$ & $\mathrm{MRG}^{*}$ \\
\hline A754 & 0.054 & $8.544 \pm 0.102$ & 2.27 & $0.5289 \pm 0.0120$ & $\mathrm{NCC}$ & MRG \\
\hline A3266 & 0.055 & $7.351 \pm 0.081$ & 2.10 & $0.6772 \pm 0.0170$ & $\mathrm{NCC}$ & $\mathrm{MRG}^{*}$ \\
\hline A2256 & 0.057 & $5.709 \pm 0.0763$ & 1.85 & $0.6300 \pm 0.0197$ & $\mathrm{NCC}$ & MRG $^{*}$ \\
\hline
\end{tabular}

Notes. ${ }^{(*)}$ Properties and the references for the clusters not included in the sample in Paper I. A496: CC and no evidence for a major merger (Tamura et al. 2001, and references therein). A478: CC and no evidence for a major merger (Sanderson et al. 2005, and references therein). PKS0745-191: $\mathrm{CC}$ and no evidence for a major merger (Chen et al. 2003, and references therein). A3532: INT (moderate temperature drop) and no evidence for a major merger (the indications of optical substructures, Bardelli et al. 2000, are insufficient to claim a major merger). A3667: NCC and MRG (evidence of radio relics Rottgering et al. 1997; optical substructures Owers et al. 2009; and disturbed thermodynamical maps Briel et al. 2004; Vikhlinin et al. 2001). A3266: NCC and MRG (even if extended radio emission has not been detected, Buote 2001, there are evidences of optical substructures; Quintana et al. 1996; and disturbed thermodynamical maps Finoguenov et al. 2006). A2256: NCC and MRG (radio halo and relic, Kim 1999, optical substructures; Berrington et al. 2002, and disturbed temperature maps; Sun et al. 2002; Bourdin \& Mazzotta 2008).

\section{Classification schemes}

As in Paper I we plot the temperature ratios $\left(T_{\mathrm{IN}} / T_{\text {OUT }}\right)$ versus the emission measure ratios $\left(E M_{\mathrm{IN}} / E M_{\mathrm{OUT}}\right)$ for the clusters of our sample (Fig. 1), and we use pseudo-entropy ratios to divide clusters into two classes (Table 2). We recall here the definition of the pseudo-entropy ratio ${ }^{3}$ :

$\sigma \equiv \frac{s_{\mathrm{IN}}}{s_{\mathrm{OUT}}}=\frac{T_{\mathrm{IN}}}{T_{\mathrm{OUT}}}\left(\frac{E M_{\mathrm{IN}}}{E M_{\mathrm{OUT}}}\right)^{-1 / 3}$.

\footnotetext{
${ }^{3}$ X-ray astronomers usually define as "entropy" the quantity $S \equiv$ $T_{\mathrm{X}} n_{\mathrm{e}}^{-2 / 3}$, where $n_{\mathrm{e}}$ is the electronic density and $T_{\mathrm{X}}$ the deprojected temperature of the ICM (Ponman et al. 1999). In this paper, we use the definition of "pseudo-entropy" $s \equiv T *(E M)^{-1 / 3}$, where $T$ is the projected temperature and $E M$ is the emission measure, i.e. the normalization of the MEKAL model in XSPEC per units of area (see Rossetti et al. 2007, for more details).
}

The pseudo-entropy ratio is well correlated with the entropy ratio (see Appendix A), and therefore it is a useful and easyto-calculate indicator of the variation of the physical threedimensional entropy in the cores of galaxy clusters.

As in Paper I, we compare our pseudo-entropy classification with two alternative classification schemes, based on the core properties (Col. 6 in Table 2) and on the dynamical state (Col. 7 in Table 2). More specifically, in Col. 6 we divide clusters into three classes: cool core (CC), intermediate systems (INT) and non-cool core (NCC), where $\mathrm{CC}$ feature a prominent surface brightness peak and a temperature gradient, NCC possess neither of these properties, while INT show only one of these observational features. In Col. 7 we divide clusters into two classes: major mergers (MRG) and clusters showing no evidence of a major merger (NOM). We consider as evidence of a major merger the presence of cluster-wide diffuse radio emission, multi peaked velocity distribution of galaxies and significant irregularities on the surface brightness and temperature maps. The lack of these 


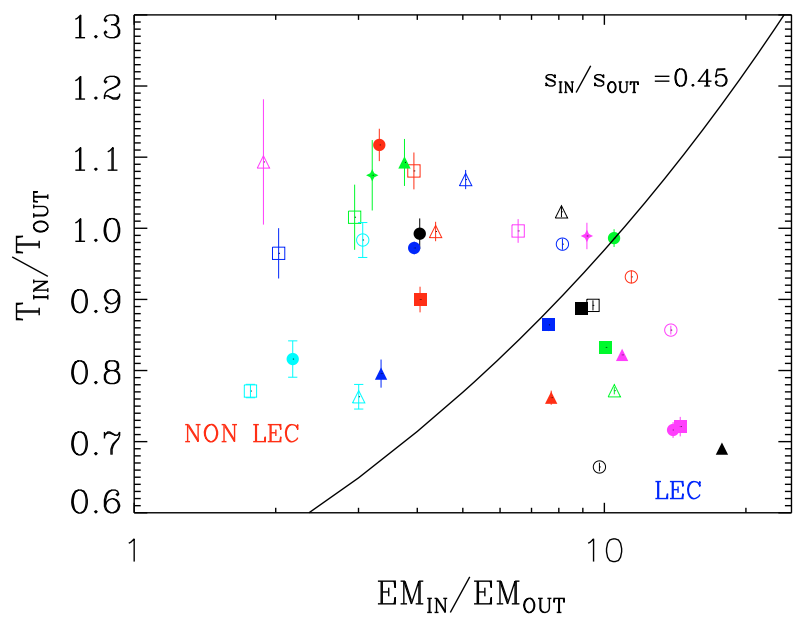

Fig. 1. Comparison of temperature and emission measure ratios for the clusters in our sample. The solid line represents the threshold used to divide clusters between low entropy clusters and non-low entropy clusters, corresponding to $s_{\mathrm{IN}} / s_{\mathrm{OUT}}=0.45$. The symbols and colors are as in Table 1.

properties is not sufficient to state that an object is relaxed, this is why we prefer to consider these objects as "not observed mergers". We refer to Paper I for more details on the classification schemes and also for the necessary references.

For the purposes of this paper, we are interested in separating clusters with a low entropy core (LEC) from the rest, this is why we have defined only one threshold to divide LEC from non-LEC objects. With our selected threshold $\left(s_{\text {IN }} / s_{\text {OUT }}=0.45\right)$ we can state that all LEC clusters are known to be CC and do not show any evidence of a merger (except A85, probably undergoing a merger in an early stage, which has not affected the observational properties of the core, see Paper I). Non-LEC clusters are almost all non-cool core systems, and many of them show significant indications of a major merger. As already discussed, the fact that some of them do not show significant merger features does not mean that they are relaxed.

\section{Cool core remnants}

The central regions of LEC clusters in our sample are characterized by low-entropy (by definition), often accompanied by a temperature decrease and a surface brightness excess. These regions are also characterized by a high metal abundance: the mean iron abundance in the cores of LEC clusters is significantly larger than the typical value of outer regions of galaxy clusters $Z=0.23 Z_{\odot}$ (Leccardi \& Molendi 2008a), even if with $\mathrm{a} \simeq 20 \%$ scatter (De Grandi \& Molendi 2009). It has been shown that the excess abundance can be entirely produced by the BCG galaxy that is invariably found in these systems (De Grandi et al. 2004). At present, we are not aware of any galaxy cluster with central regions characterized by a low entropy gas without a metal abundance excess. A close relation between entropy and metal abundance must consequently exist and also generally speaking between the thermodynamics and the chemical properties of the ICM. An example of this relation can be seen in Fig. 2, where we plot the metal abundance radial profile as a function of the pseudo-entropy ratio for each LEC cluster. The pseudo entropy ratio is similar to the one defined in Eq. (2), with the one difference that the temperature and emission measure of a given annulus replace $T_{\mathrm{IN}}$ and $E M_{\mathrm{IN}}$, i.e. $s(r) / s_{\mathrm{OUT}}=$ $T(r) / T_{\mathrm{OUT}} *\left(E M(r) / E M_{\mathrm{OUT}}\right)^{-1 / 3}$. In the lower panel of Fig. 2, we plot the mean error weighted abundance profile as a function of the pseudo-entropy ratio for the LEC clusters and the one $\sigma$ scatter around the average of the values. Low entropy ICM is characterized by a a significant metal abundance excess, although the scatter is quite large for $s / s_{\text {out }}<0.4$. The plots show that all LEC clusters show a significant metal abundance excess with respect to the outer mean value $Z=0.23 Z_{\odot}$ (Leccardi $\&$ Molendi 2008a) in regions characterized by a pseudo entropy ratio smaller than 0.8 (corresponding to physical radii $\left.r \lessgtr 0.07 R_{180}\right)$.

In Paper I we found that some clusters without a low-entropy core presented an unusually high metal abundance in their IN region (as already discussed, such high abundances are typical of LEC). We concluded that the most likely explanation for the high central abundance of these anomalous non LEC systems was that at some time in the past they hosted a cool core (i.e. lowentropy gas and metal abundance excess) that was subsequently heated up. Indeed, a heating event that does not completely disrupt a cool core will most likely leave behind a region characterized by a high metalicity and by an entropy that, albeit not as low as the one found in the central regions of LEC systems, will be lower than that found in other regions of the cluster. We emphasize that metals are reliable markers of the ICM in the sense that once they have polluted a given region of a cluster, the time scale over which they will diffuse is comparable to the Hubble time (Sarazin 1988). Thus metals trace the ICM where they have been injected. The presence of these regions has also been predicted by the simulations of Motl et al. (2004), who suggested that even if the observational cool core signature may disappear, cool gas (and we may add "low-entropy and metal rich gas") remains in the merging systems at all times.

Since in LEC clusters a metal abundance excess is invariably associated with ICM with a low pseudo-entropy, we have systematically selected the regions with the lowest pseudo-entropy ratio in non-LEC systems, with the aim of finding regions possibly characterized by a high metalicity. In practice we have prepared two-dimensional pseudo-entropy ratio maps for the 21 non-LEC clusters of our sample, by dividing the pseudoentropy maps for the pseudo-entropy in the OUT region derived with spectral analysis. In these maps we have identified regions characterized by a $s / s_{\text {OUT }}<0.8$ (as shown in Fig. 2 all LEC clusters show a metal abundance excess in regions with entropy ratio $<0.8$ ), and we have extracted and analyzed spectra in these regions (as described in Sect. 2.4.1) to investigate their metalicity. Pseudo-entropy ratio maps and figures of the selected regions are provided in Appendix B.

In all the objects (21) of our subsample of non-LEC clusters, we find regions characterized by an entropy ratio smaller than 0.8. In Fig. 3 we plot the metal abundance in these regions for all the clusters (results are reported in Table 3). $12 \mathrm{ob}-$ jects (namely: A1689, MKW3s, A4038, A1650, A1644, A3558, A576, A754, A3562, A3571, A3667 and A2256) show a significant excess ( $>3 \sigma$ c.l.) with respect to the reference value $Z=0.23 Z_{\odot}$ (Leccardi \& Molendi 2008a). In the remaining nine clusters, the metal abundance is statistically consistent with the reference value. Amongst these objects, we will consider as clusters without metal abundance excess those where the metalicity is measured with a good statistical accuracy, more specifically the six clusters where $Z>0.4 Z_{\odot}$ can be excluded at a confidence level $>2 \sigma$ (A2319, A3266, A399, A401, A119 and A3158). We will no more consider in this paper the three objects (A3532, Triangulum Australis and A2255) where the error bars on the metal abundance are too large to discriminate between the two 

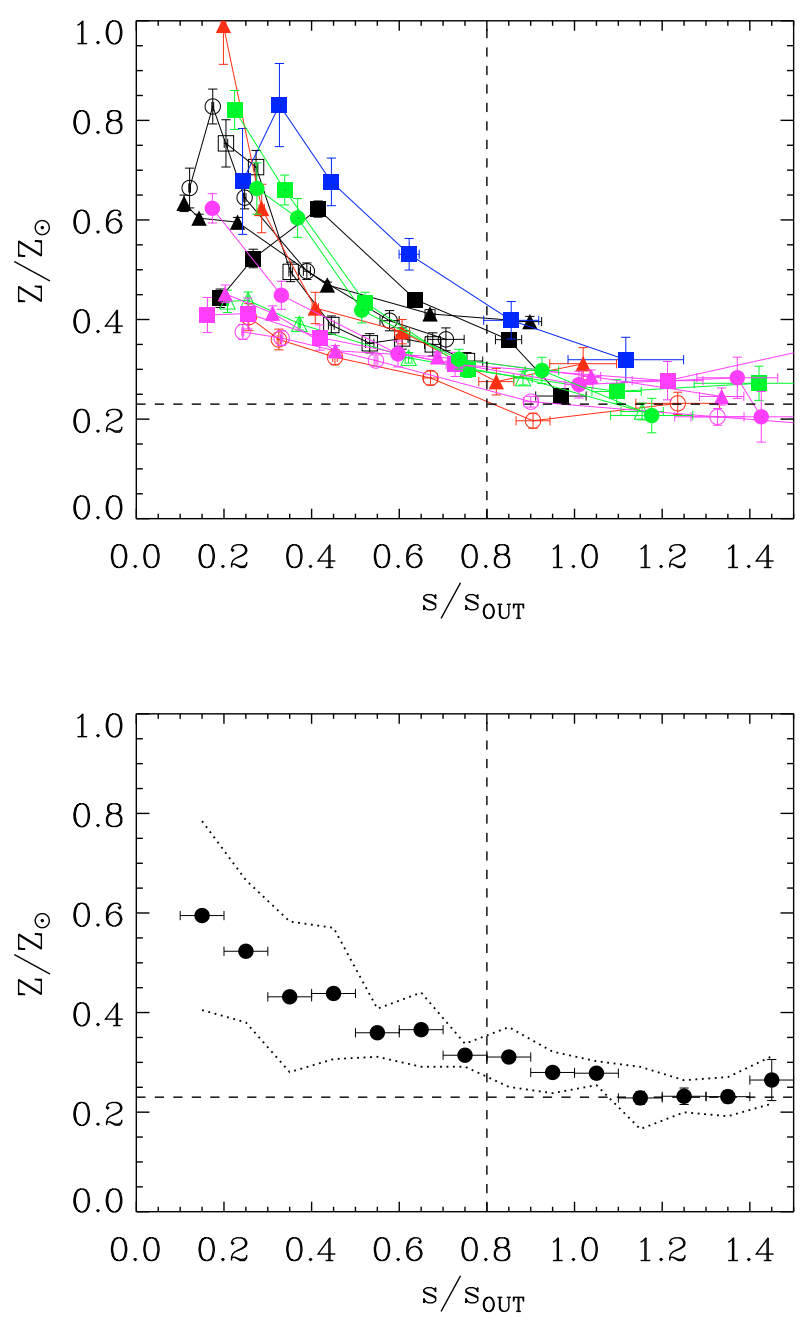

Fig. 2. Upper panel: metal abundance profiles of LEC clusters as a function of the pseudo-entropy ratio, in the regions of spectral extraction. The symbols and colors are as in Table 1. Lower panel: mean error weighted abundance profile for LEC clusters. Error bars show the (small) error on the average, while dotted lines show the $1 \sigma$ scatter around the mean. In both panels, the horizontal dashed line indicates the mean value of outer regions of galaxy clusters $Z=0.23 Z_{\odot}$ (Leccardi $\&$ Molendi 2008a) and the vertical dashed line indicates the selected threshold of $s / s_{\text {OUT }}$ under which all LEC cluster show a significant metal abundance excess.

classes (the observations of these clusters are the shortest of our sample, see Table 1).

The most likely interpretation is that the low entropy high $Z$ regions found in many of our non-LEC systems are what remains of a cool core after a heating event. We dub these structures "cool core remnants" , a more detailed discussion of how they formed and of alternative scenarios is provided in Sect. 5.

In the lower panel of Fig. 3 we plot the metal abundance as a function of the pseudo entropy ratio $s / s_{\text {OUT }}$ where $s$ is the pseudo-entropy in the region used for spectral analysis. It is worth noting that the clusters with no indication of a significant metal abundance excess are those where the selected regions show the highest $s / s_{\text {OUT }}$, with the notable exception of A2319 (red filled square).

\footnotetext{
4 The term "remnant of cool cores" has been recently used by Fusco-Femiano et al. (2009) to describe structures similar to the ones we detect albeit without information on the metal abundance.
}
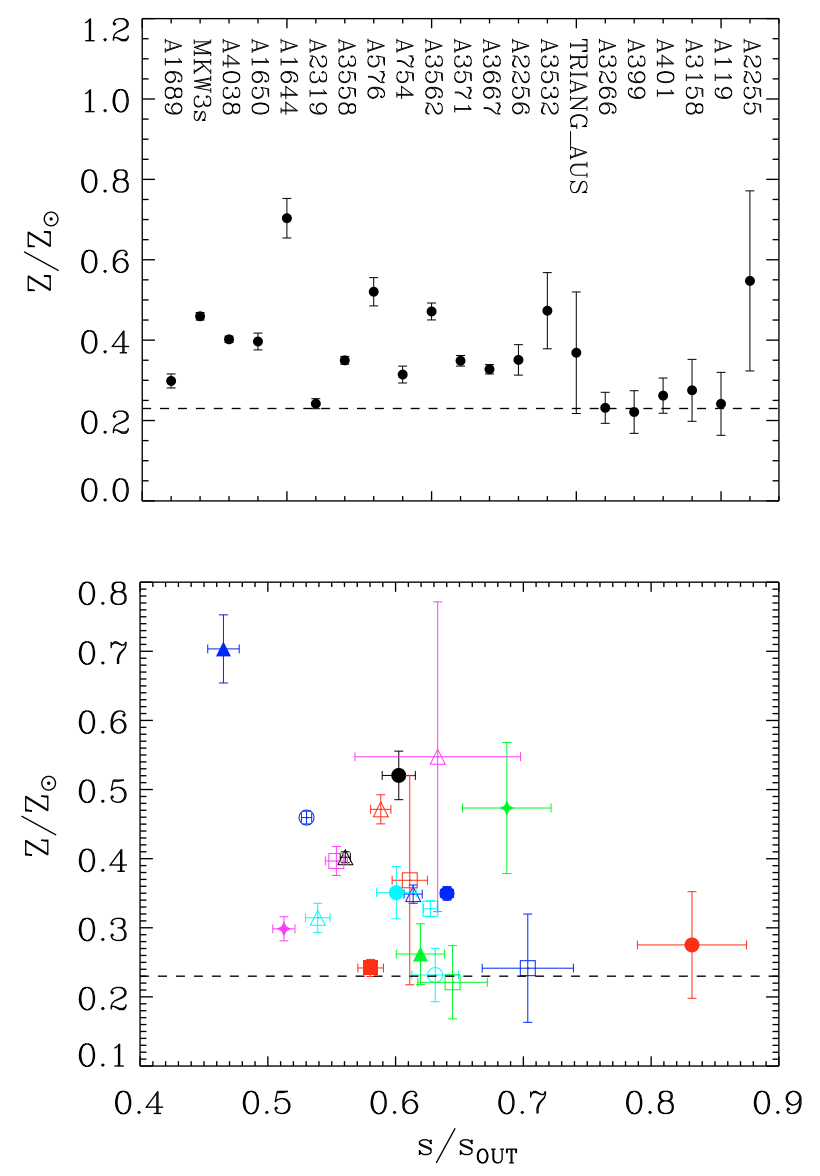

Fig. 3. Metal abundance in the regions selected for spectral analysis for the 21 non-LEC objects in the sample (upper panel) and as a function of the pseudo-entropy ratio in the regions (lower panel). The horizontal line represents the reference value for the metalicity of outer regions of galaxy clusters $Z=0.23 Z_{\odot}$ (Leccardi \& Molendi 2008a). Symbols and colors are as in Table 1.

To better characterize the systems in our sample, we have compared the entropy maps with the galaxy distribution and more specifically the low-entropy regions with the position of the BCG (Col. 3 in Table 3 and figures in Appendix B). The name and positions of the BCGs are derived from the literature (Coziol et al. 2009; Lin \& Mohr 2004; Gudehus \& Hegyi 1991; Postman \& Lauer 1995). In 9/12 clusters with "CC remnants" (namely A1650, MKW3s, A4038, A1644, A3558, A3562, A3571, A1689 and A576) the BCGs are located in the low-entropy high metal abundance regions. In A3667 and A2256, the positions of the BCGs do not coincide with the "CC remnant", which is moreover not obviously associated to any galaxy concentration (a more detailed study of the galaxy distribution in A3667 by Owers et al. 2009, found essentially the same results). In A754 the BCG is located $700 \mathrm{kpc}$ west of the selected regions, but in the "CC remnant" we find the elliptical galaxy 2MASSX J09091923-0941591 (see figure in Appendix B), which is the brightest member of the secondary peak in the galaxy distribution (Fabricant et al. 1986). The position of this secondary galaxy concentration, possibly associated to the $\mathrm{CC}$ remnant, coincides also with a clump in the dark matter distribution, as derived with gravitational lensing (Okabe \& Umetsu 2008). For the non-LEC objects where we do not observe a significant metalicity excess, we find that in 3/6 systems (A119, A401 and A3158) the low entropy regions are not obviously associated to the BCGs. In the remaining 3 clusters (A399, 
Table 3. Properties of low entropy regions: clsters in Italics are "AGN CC-remnants", while clusters in bold type are "merger CC-remnants".

\begin{tabular}{|c|c|c|}
\hline Cluster & Metal abundance (solar) & $\mathrm{BCG}^{a}$ \\
\hline A1650 & $0.397 \pm 0.021$ & $\mathrm{Y}$ \\
\hline$M K W 3 s$ & $0.459 \pm 0.009$ & $\mathrm{Y}$ \\
\hline$A 4038^{b}$ & $0.402 \pm 0.008$ & $\mathrm{Y}$ \\
\hline$A 1644^{b}$ & $0.703 \pm 0.049$ & $\mathrm{Y}$ \\
\hline A1689 & $0.298 \pm 0.017$ & $\mathrm{Y}$ \\
\hline A3558 & $0.350 \pm 0.010$ & Y \\
\hline A576 & $0.520 \pm 0.035$ & $\mathrm{Y}$ \\
\hline A754 & $0.314 \pm 0.021$ & $\mathrm{~N}^{c}$ \\
\hline A3562 & $0.471 \pm 0.021$ & $\mathrm{Y}$ \\
\hline A3571 & $0.349 \pm 0.013$ & Y \\
\hline A3667 & $0.327 \pm 0.012$ & $\mathrm{~N}$ \\
\hline A2256 & $0.351 \pm 0.038$ & $\mathrm{~N}$ \\
\hline A2319 & $0.242 \pm 0.012$ & $\mathrm{Y}$ \\
\hline A3266 & $0.232 \pm 0.039$ & Y \\
\hline A399 & $0.221 \pm 0.053$ & $\mathrm{Y}$ \\
\hline A401 & $0.262 \pm 0.044$ & $\mathrm{~N}$ \\
\hline A3158 & $0.275 \pm 0.077$ & $\mathrm{~N}$ \\
\hline A119 & $0.242 \pm 0.078$ & $\mathrm{~N}$ \\
\hline $\mathrm{A} 2255^{d}$ & $0.547 \pm 0.224$ & \\
\hline $\mathrm{A} 3532^{d}$ & $0.473 \pm 0.095$ & \\
\hline Triangulum Australis $^{d}$ & $0.369 \pm 0.151$ & \\
\hline
\end{tabular}

Notes. (a) "Y" if the BCG is found in the low-entropy region and "N" if it is elsewhere. ${ }^{(b)}$ even if the central entropy of this cluster is within the reach of AGN outbursts, it also shows some indications of on-going interaction and cannot be unambiguously associated to the "AGN CC-remnant class" (see text). ${ }^{(c)}$ The BCG 2MASSX J090832380937470 is not associated to the CC-remnant, which however coincides with the position of the elliptical galaxy 2MASSX J09091923-0941591.

(d) Due to the large indetermination of the metal abundance estimate, we could not unambiguously detect or exclude the presence of a metal abundance excess in this object.

A3266, and A2319) the BCGs are located in the low-entropy regions where we do not find evidence of a metal excess.

The main reason why we compared the position of $\mathrm{CC}$ remnants with the galaxy distribution is that galaxies, and more specifically BCGs, are responsible for the metal abundance excess observed in LEC clusters (De Grandi et al. 2004). However, the galaxy distribution may also provide information on the shape of the potential well of the clusters. Indeed, in relaxed clusters BCGs are located at the bottom of the potential well and their positions coincide with the centroid of the X-ray isophotes and with the brightness peak of their host clusters. We have compared the position of CC remnants with the position of the X-ray peak, the centroid of X-ray isophotes and the position of the BCGs (figures in Appendix B). In most cases (namely A1650, MKW3s, A4038, A1644, A1689, A576, A3562, and A3571), these three points lie within the low entropy regions even if they do not necessarily coincide with one another. However, in the remaining clusters one or more of these indicators is significantly offset from the CC remnant. A754 and A2256 have a very disturbed morphology, and the X-ray centroid does not coincide with the CC remnant or with the BCG. In A3667 the centroid roughly coincides with the brightness "peak" and the BCG, but the CC remnant is offset by almost $500 \mathrm{kpc}$. It is apparent that in these last three cases the low entropy metal rich regions are not in equilibrium within the cluster's potential well, and therefore we are observing a rapidly evolving situation.

\section{Discussion}

We have shown in Sect. 4 that 12 out of 21 non-LEC objects of our sample host regions characterized by low entropy and high metal abundance with respect to the mean values in the outer ICM. Such conditions are usually verified in the cores of relaxed LEC clusters, albeit with stronger gradients (i.e. the entropy of the ICM reaches smaller values). We have dubbed these features "cool core remnants", since we have interpreted them as the remains of a cool core after a "heating event".

One may argue that these structures have evolved similarly but independentely of LEC systems and that there is no need to trace them back to known structures such as LEC. In the "primordial" scenario, where non-LEC clusters started off with different early conditions and are now slowly cooling to become LEC, the low entropy and metal-rich regions could be interpreted as "progenitors" of cool cores, rather than as remnants. However, this scenario does not explain why in some cases these structures are not in equilibrium within the potential well of their host clusters and are not associated to the BCG or to a giant elliptical galaxy. On the contrary, these issues are naturally addressed in the "evolutionary" scenario if we consider the effects of mergers both on the ICM and on the galaxy distribution (Sect. 5.2).

Moreover, we should pay attention to the fact that most of these structures are found in clusters undergoing major mergers, i.e. in rapidly evolving objects. It is therefore necessary to trace these transient structures back to an equilibrium state from which they recently evolved.

Another possible objection is that the low-entropy structures may not be embedded in the ICM but rather in groups along the line of sight of the clusters. However, in the cases where the cool core remnants are associated to the BCGs or to a galaxy concentration (see Sect. 4), the redshifts of the galaxies are consistent with the mean readshift of the clusters. In the cases where there is no galaxy concentration obviously associated to the low entropy regions, these features would have to be interpreted as "gas-only" groups superposed on the line of sight.

In the interpretation of the low-entropy regions as $\mathrm{CC}$ remnants, the "heating event" is responsible for smoothing the entropy gradient of the cores of LEC clusters. We have identified two possible mechanisms: interaction with the central AGN and mergers.

\subsection{CC remnants and central AGNs}

Interactions between the central AGN and the ICM have been observed with Chandra and XMM-Newton in many LEC clusters, and these interactions are now considered as the principal mechanism preventing the formation of cooling flows (Peterson $\&$ Fabian 2006, and references therein). In some cases, powerful AGN outbursts may significantly increase the entropy content of some galaxy clusters, transforming a low entropy core into a non-LEC object. This is possible for systems where the core entropy has been raised to a relatively small value $s_{0} \simeq(30-50) \mathrm{keV} \mathrm{cm}^{2}$ (Voit \& Donahue 2005). In our subsample of non-LEC clusters according to Chandra measurements (Cavagnolo et al. 2009) only A1650, A4038, MKW3s and A1644 have a core entropy smaller than $50 \mathrm{keV} \mathrm{cm}^{2}$ and are therefore within the reach of powerful AGNs. AGN heating is the most plausible explanation for increasing the entropy in the cores of A1650 (Donahue et al. 2005; Voit \& Donahue 2005) and for MKW3s (radio lobes have been observed in this cluster, Giacintucci et al. 2006). We will consider these clusters as "AGN CC-remnants". 


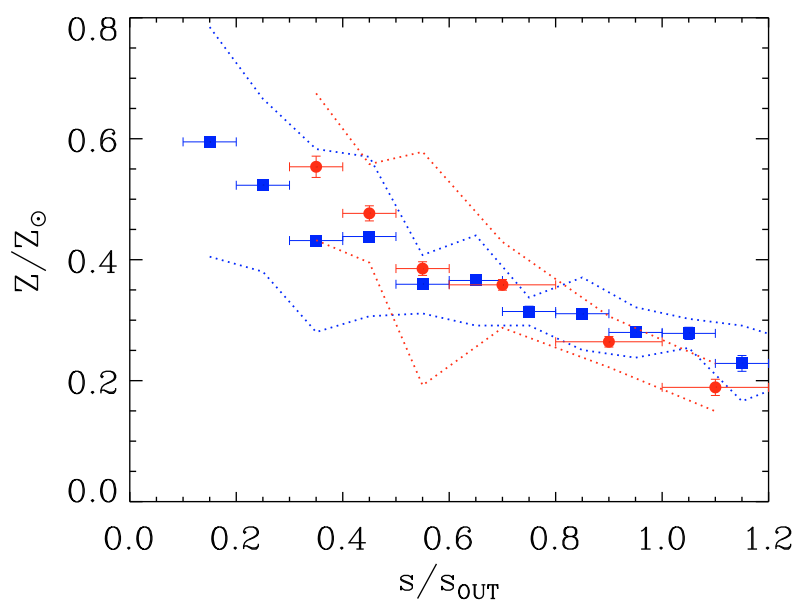

Fig. 4. Error weighted metal abundance profile as a function of the pseudo entropy ratios for LEC clusters (blue squares) and for the four clusters with low central entropy (red circles). Dotted lines show the one $\sigma$ scatter of the data.

The remaining two non-LEC clusters with a relatively low central core entropy, A4038 and A1644, are controversial objects, showing some indications of ongoing interactions contrary to A1650 and MKW3s, which look relaxed at all wavelengths (see Table 2 in Paper I). A4038 does not have a large temperature drop at the center, but it has a low central cooling time. This is why some authors classify it as a cool core (Peres et al. 1998) and others as a non-cool core (Sanderson et al. 2006). In the optical, Burgett et al. (2004) found some indications of substructure on large scales. A1644 is probably an advanced off-axis merger with two subclumps clearly visible in the X-ray image and some indications of on-going sloshing of the cool core of the main subclump (Reiprich et al. 2004). The interpretation of A4038 and A1644 is not straightforward, since merging events could have contributed to the heating of the cores (see Sect. 5.2).

In AGN CC remnants, the low entropy regions coincide with the centers of the clusters and with the positions of the BCGs as expected. In Fig. 4 we compare the error weighted mean metal abundance profile of LEC clusters with the same profile for non-

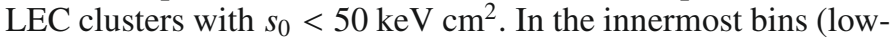
est entropy ratios), the mean profile of this class of clusters is significantly larger than in LEC clusters, although the scatter is large. This is consistent with a scenario where a localized heating in the inner regions of clusters has increased the entropy ratio without substantially modifying the metal abundance, resulting in ICM with enhanced metalicity for its entropy.

\subsection{CC remnants and mergers}

As shown in Sect. 5.1, eight out of 12 clusters featuring a metal abundance excess have core entropies too large to be produced even by the most powerful AGN outbursts. Therefore, we need another mechanism which could have produced the CC-remnants that we observe today. The fact that most of these clusters (namely A754, A3667, A2256, A3562 and A576) show strong indications of a major merger suggests that merging could provide the necessary heating to produce these features. The indications are not as strong for the remaining three clusters (A1689, A3558 and A3571; in Table 2 they are classified as "intermediate" and "no observed merger"). However, as shown in the notes reported below, they all possess some peculiar features, which suggest that they are undergoing some kind of interaction. It is therefore natural to define this class of eight objects as "merger CC remnants".

\section{Notes on A1689, A3558 and A3571}

A1689 This cluster has been considered for a long time by $\mathrm{X}$-ray astronomers as an example of a relaxed cluster due to its spherical shape (figure in Appendix B) and very peaked surface brightness profile (e.g. Peres et al. 1998). However, analysis of the distribution of the velocities of the galaxies showed two distinct peaks, suggesting a line of sight superposition (Girardi \& Mezzetti 2001). More recently, an analysis of XMM-Newton data by Andersson \& Madejski (2004) showed asymmetric temperature and redshift distribution, which, combined with optical data, could suggest an on-going merger.

A3558 Rossetti et al. (2007) classify this cluster as an intermediate object, since it has some characteristics of $\mathrm{CC}$, but it also shows some indications of an on-going merger, like asymmetry in the temperature and entropy distribution and substructures in the 3D galaxy distributions (Bardelli et al. 1998). Due to the very peculiar environment in which it is located (the core of the Shapley Supercluster), these features may be explained as the results of multiple minor mergers or of a past off-axis major merger with A3562.

A3571 This cluster has a regular morphology in X-rays (figure in Appendix B), but we do not observe a temperature decrease in the center. This is why it is often classified as a "non cool core" object (O'Hara et al. 2006; Sanderson et al. 2006). On the basis of its radio and optical properties, Venturi et al. (2002) suggest that this cluster is a late stage merger.

We recall here the main results concerning the position of the merger CC remnants with respect to the centroid of X-ray emission and to the galaxy distribution (Sect. 4 and Table 3). In 5/8 clusters (namely A3558, A3562, A3571, A1689, A576) the BCGs and the X-ray centroid are located in the low-entropy high metal abundance regions. In A754 the CC remnant is associated with a secondary peak in the galaxy distribution (with a giant elliptical galaxy), but it is also offset from the position of the large scale X-ray centroid. In A3667 and A2256 the CC remnants are not obviously associated with any galaxy concentration and they are not found at the centers of their host clusters (see figures in Appendix B). These different results do not contradict our interpretation since the effect of the merging processes both on the collisional ICM and on the non-collisional dark matter and galaxies depends on many parameters such as the merger state, the mass-ratios and the impact parameter. Therefore, while in A3667 and A2256 the low entropy and high metal abundance gas may have completely decoupled from its galaxy concentration and possibly dark matter halo (such as in the "bullett cluster", Clowe et al. 2006), in A3558, A3562, A3571, A1689, A576 and A754 this gas has not been completely displaced from its gravitational potential well. Another possibility is that we are currently observing a late stage of a merger in the latter systems, where the low entropy high metal abundance gas is slowly moving back to the center of the cluster.

Two main mechanisms can contribute to the formation of a CC remnant during a merger event: shock-heating and mixing. Mergers are expected to drive moderately supersonic shock waves in the ICM, which, being irreversible changes, increase the entropy of the system. If one of the merging subclusters had a $\mathrm{CC}$ before the merging event, shock-heating may significantly 


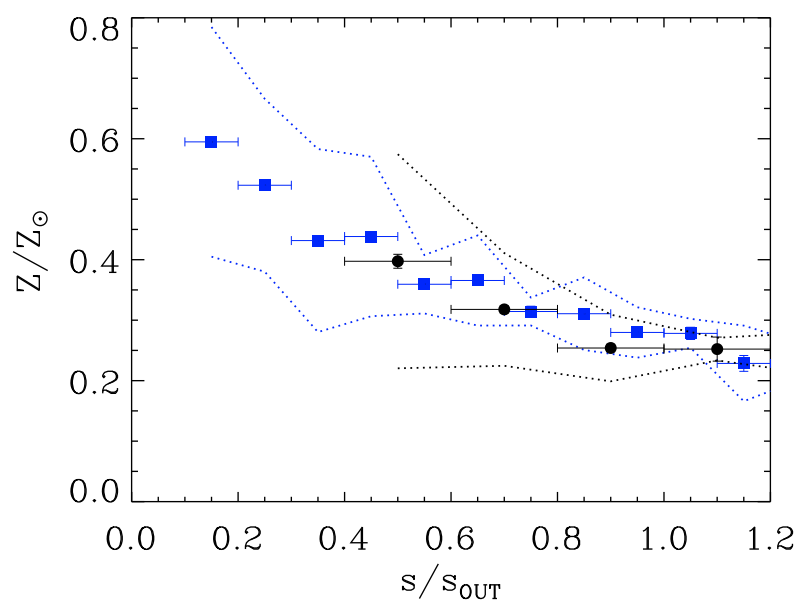

Fig. 5. Error weighted metal abundance profile as a function of the pseudo entropy ratios for LEC clusters (blue squares) and for the clusters with merger CC remnants (black circles). Dotted lines show the one $\sigma$ scatter of the data.

increase the entropy of the core, but in some cases this heating may not be sufficient to reach the entropy values observed in some non-LEC clusters. With a very simplified calculation based on Rainkine-Hugoniot jump conditions, we estimate that shocks with a Mach number of 1-3 can increase the mean entropy in the cores only to a factor of up to 1.8. Mergers also drive motions in the ICM in which low entropy metal-rich gas may be put in contact with high-entropy metal poor ICM. The mean entropy of the resulting mixed ICM will be lower than the ambient entropy (but higher than the typical core entropy), and its metal content could be larger than in the ambient gas. However, since there is a significant spread in the metal abundance values of cool cores, mixing may also efficiently disrupt small abundance gradients. We recall here that metals are frozen in the ICM, and therefore mixing with metal poor ICM is the only possible way of reducing the metal abundance.

In Fig. 5 we compare the mean metal abundance profile for clusters with merger CC remnants with that of LEC clusters, as in Fig. 4 for AGN CC remnants. In this case the mean profiles are consistent but the scatter in clusters with CC remnants is larger. Indeed, we did not expect the same results as in Fig. 4 (i.e. gas with enhanced metalicity for its entropy) because, contrary to AGN heating, merger heating does not affect only the central regions of clusters and therefore the entropy ratios are modified in a non-trivial way. Moreover, we cannot neglect the effect of gas mixing, both on the entropy and on the metal abundance.

\subsection{Non LEC clusters without metal abundance excess}

Of the 21 objects of our sample which are classified as non LEC, six feature regions with pseudo-entropy ratios smaller than 0.8 , but without a significant metal abundance excess $\left(Z<0.4 Z_{\odot}\right.$ at more than $2 \sigma$ confidence level). We will discuss in this section some of the properties of these systems.

As shown in Table 2, we have strong indications of ongoing mergers for five out of six objects, namely A2319, A399, A401, A119 and A3266. The remaining cluster, A3158, shows some indications of ongoing interactions based mainly on the galaxy distribution (Johnston-Hollitt et al. 2008). These clusters do not have a CC remnant, and this could mean that they have never developed a cool core, as in the scenario proposed by McCarthy et al. (2008). However, we should note that while in this "primordial" scenario NCC may indifferently be relaxed or nonrelaxed objects, in most objects of this class (if not all) we have found indications of on-going interactions.

The fact that most of these clusters show indications of ongoing interactions could suggest that they may have had a lowentropy core that has been completely erased during the merger event. As shown in many simulation works, the effects of a merger on the structure of the ICM depends on many parameters, such as the mass ratio, the impact parameter and the structure of the interacting subclusters. Therefore it is not surprizing that in some cases the merger can efficiently destroy the low entropy core, while in others it leaves a CC remnant.

We recall here than in three clusters (A399, A3266 and A2319) the BCGs are located in the low-entropy region where we do not find evidence of a metal excess (see Table 3). The interpretation of this class of clusters is an interesting task: we speculate that either the LEC state from which they evolved was characterized by a metal abundance profile showing only a moderate excess or, alternatively, that the mixing may have been more effective in these clusters than in those where we observe $\mathrm{CC}$ remnants. We recall that metalicity profiles of LEC clusters show a large scatter: as can be seen also in Fig. 2, while the central metal abundance of some clusters can reach almost solar values, in others it reaches only $0.4 Z_{\odot}$. Assuming a constant degree of mixing in all clusters, it is clear that it would be easier to detect a metal excess in regions where gas with $Z \sim Z_{\odot}$ has mixed with the ambient gas than in regions where mixing has occurred between ICM with $Z \simeq 0.4 Z_{\odot}$ and ICM with $Z \simeq 0.2 Z_{\odot}$. Therefore systems like A399, A3266 and A2319 could be remnants of "metal poor CC", where "poor" means that the metal abundance is lower with respect to other CC objects. Alternatively, these could be systems where metals have been mixed more efficiently than in others where an abundance excess is detected.

It is interesting to note that the fraction of objects where the BCG is not associated to the low-entropy regions is higher in clusters showing no $Z$ excess $(3 / 6)$ than in merger $C C$ remnants $(2 / 8)$, even if we should note that this difference is not statistically significant. As shown in many simulations and in the case of the "bullett cluster" (Clowe et al. 2006), in violent major mergers the collisionless galaxy population may completely decouple from the collisional ICM. It is natural to assume that these violent mergers are also more effective in mixing the gas and therefore in completely erasing metal abundance gradients. In this scenario, the three clusters showing no metal abundance excess and no BCG associated, namely A119, A3158 and A401, should be those that have undergone the most destructive interactions. However, this speculation needs further verification with a larger sample of clusters.

\subsection{Metal abundance and entropy distribution}

One may speculate whether our selection based on the pseudoentropy ratio $<0.8$ is effective in identifying all the regions with a large metalicity. Indeed, a possible counter-example to our choice of using an entropy ratio threshold for the selection of regions for spectral analysis would be the observation of a large metal abundance associated with high entropy gas, and generally speaking the lack of anti-correlation between metal abundance and pseudo-entropy ratio.

In Fig. 2 we have shown that the metal abundance excess in LEC clusters is typically found in regions with $s / s_{\text {OUT }}<$ 0.8. In a few cases, an excess is detected also in regions with 


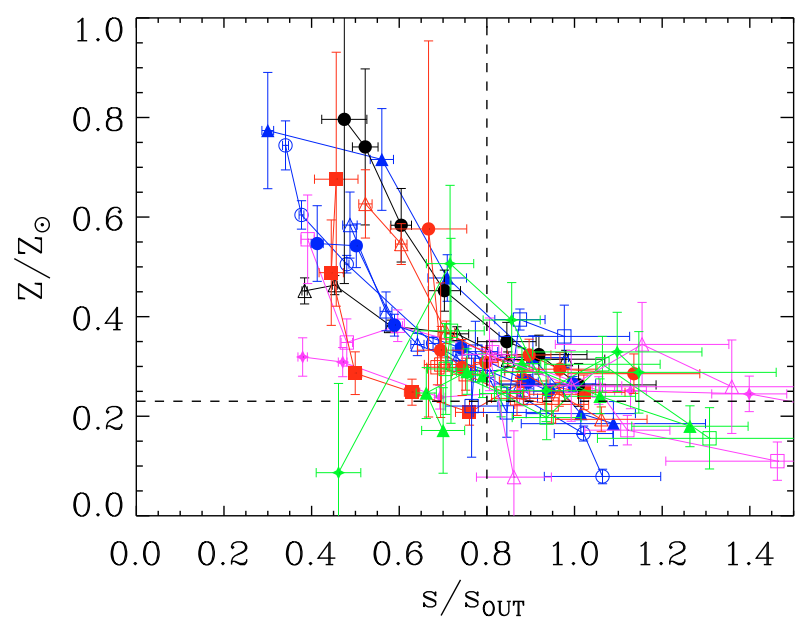

Fig. 6. Metal abundance profiles of non-LEC clusters as a function of the pseudo-entropy ratio in the regions of spectral extraction. The symbols and colors are as in Table 1. The dashed horizontal line indicates the mean value of outer regions of galaxy clusters $Z=0.23 Z_{\odot}$, (Leccardi \& Molendi 2008a), while the vertical dashed line marks the threshold pseudo- entropy ratio used to select regions for spectral analysis.

$s / s_{\text {OUT }} \sim 1$, but not at larger values. Moreover, the metal abundance is always anti-correlated with the entropy.

We have performed the same test on the subsample of nonLEC clusters, using the results of spectral analysis in radial annuli centered on the surface brightness peak (Fig. 6). We do not include in this figure the four highly disturbed clusters for which we have not performed spectral analysis in radial annuli. It is interesting to note that we do not find a single region where a significant metal excess is associated to a pseudo entropy ratio larger than 1. If a metal abundance excess is observed, it is invariably associated with ICM with a low pseudo-entropy ratio.

\subsection{Cooling times}

The results presented in this paper support the "evolutionary" scenario of the CC-NCC dichotomy: most non-LEC clusters have likely "evolved" from a LEC state. It is therefore natural to ask ourselves if this is a "one way trip", i.e. whether once a cluster has undergone a merger it remains a non-LEC cluster for the rest of its life or it has the possibility of reforming a low-entropy core, as in the old cyclic vision of cooling flows-mergers.

In Fig. 7 we plot the cooling time in a central bin of a radius $0.05 R_{180}$ for the clusters of the initial sample for which we have performed radial analysis and de-projection (i.e. excluding A3266, A2256, A3667 and A754). The cooling time is generally larger than the Hubble time for non-LEC clusters, including those of most clusters with a CC-remnant (A576, A3571, A3562, A3558, we do not have this 3D information for A3667, A754 and A2256). Therefore these clusters would appear to be unable to develop a new low-entropy core.

The cooling time shown in Fig. 7 is a mean value calculated in a large region. If the mixing of the ICM is not completely effective, clumps of cool gas may reside in these regions, and in these clumps the cooling will be much more effective. In this case, we should consider the measured cooling time only as an upper limit, and it is possible that the cooler gas could contribute to the formation of a low entropy core on time scales shorter than the Hubble time. Any future observation of these clumps of cool

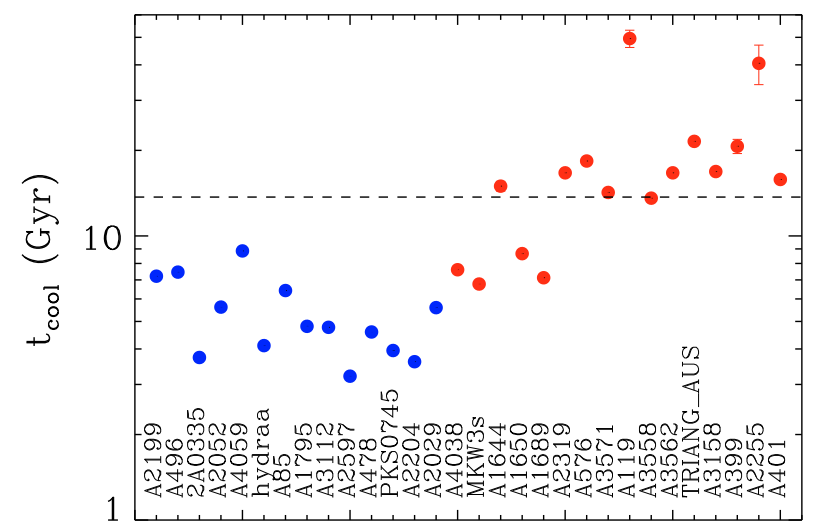

Fig. 7. Cooling time in the inner $0.05 R_{180}$ for the clusters of the sample for which we have performed radial analysis and de-projection. Horizontal dashed line is the Hubble time.

gas will be useful to test the possibility of a "return journey" to the LEC state.

\section{Summary and conclusions}

We have presented a systematic two-dimensional analysis of the entropy and metal abundance of a sample of nearby clusters observed with XMM-Newton.

- We have analyzed the observations of a sample of 35 clusters. Following the prescription in Paper I, we have classified 14 of them as low-entropy cores. In the remaining 21 non-LEC objects we have performed a systematical analysis of the entropy maps and selected regions with a pseudoentropy ratio lower than 0.8 for a proper spectral analysis. All the clusters in our subsample host regions with these characteristics.

- In most non-LEC objects (12/21) the low entropy regions are characterized by a significant metal abundance excess with respect to outer regions of galaxy clusters. For six of the remaining clusters we can exclude with high confidence an abundance larger than $0.4 Z_{\odot}$, while for three objects the error bars are too large to discriminate between the two classes. We have interpreted the low-entropy high metal abundance regions that we found in 12 clusters as the remains of a cool core after a heating event, and we have dubbed them "CC remnants".

- For two clusters, A1650 and MKW3s, the most likely heating mechanism is AGN feedback. As shown by Voit \& Donahue (2005), clusters with a core entropy of $\$ 50 \mathrm{keV} \mathrm{cm} \mathrm{cm}^{2}$ are within the reach of powerful AGN outbursts.

- In 8/12 clusters with a CC remnant, the core entropy is too large to be produced by giant AGN outbursts. In all these clusters we have found indications of on-going interactions, and five of them are probably undergoing a major merger. Therefore the most likely heating mechanism for this class of objects is merging, which can shock-heat and mix the ICM. In most of these clusters, the CC remnant is associated with the BCG or with a giant elliptical galaxy, which could be responsible for the production of the metal excess during the LEC phase. In A3667 and A2256 the merging may have completely decoupled the low-entropy metal-rich gas from its associated galaxy concentration. In A754, A2256 and $\mathrm{A} 3667$ the position of the CC remnant does not coincide with the centroid of the X-ray emission, indicating that the 
low-entropy metal-rich ICM is not in equilibrium within the potential well of the host clusters.

- In six objects out of 21 we have not found a significant metal excess in the low entropy regions. Since most of these clusters show indications of major mergers, we have interpreted them as clusters where the metal abundance gradient has been erased by the mixing following mergers.

The results presented in this paper strengthen those shown in Paper I. Indeed, in Paper I we found a metal abundance excess in a small fraction of non-LEC clusters. Thanks to the twodimensional analysis presented in this paper, we have found a metal abundance excess in most objects. We conclude that most non-LEC objects have spent part of their life as low-entropy cores. After a heating event the LEC signature disappears, but the "CC-like" ICM (i.e. characterized by large metal abundance and low entropy, albeit not as low as in LEC) remains in the systems and can be identified in two-dimensional entropy maps. In the framework of the alternative "primordial" scenario the low entropy metal rich regions could be interpreted as "progenitors" of cool cores that are now evolving (slowly cooling and increasing their metalicity) to become LEC. However, this scenario does not explain why most of these regions lie in dynamically active objects and why at least three of them are found in a configuration of non-equilibrium, offset from the center of the clusters. Conversely, this issue is naturally addressed if they are the results of a merger event in the evolutionary scenario.

The results summarized above strongly support scenarios where cluster core properties are not fixed "ab initio", but evolve across cosmic time.

Acknowledgements. We thank Fabio Gastaldello and Sabrina De Grandi for useful discussions. We acknowledge the financial contribution from contracts ASI-INAF I/023/05/0 and I/088/06/0. This paper is based on observations obtained with XMM-Newton, an ESA science mission with instruments and contributions directly funded by ESA Member States and NASA. This research has made use of the NASA/IPAC Extragalactic Database (NED), of the High Energy Astrophysics Science Archive (HEASARC), of the XMM-Newton Science Archive (XSA), of the Digitized Sky Surveys (DSS) and of the Archive of Chandra Cluster Entropy Profiles Tables (ACCEPT).

\section{References}

Anders, E., \& Grevesse, N. 1989, Geochim. Cosmochim. Acta, 53, 197

Andersson, K. E., \& Madejski, G. M. 2004, ApJ, 607, 190

Arnaud, M., Pointecouteau, E., \& Pratt, G. W. 2005, A\&A, 441, 893

Ascasibar, Y., \& Markevitch, M. 2006, ApJ, 650, 102

Bardelli, S., Pisani, A., Ramella, M., Zucca, E., \& Zamorani, G. 1998, MNRAS, 300,589

Bardelli, S., Zucca, E., Zamorani, G., Moscardini, L., \& Scaramella, R. 2000, MNRAS, 312, 540

Bauer, F. E., Fabian, A. C., Sanders, J. S., Allen, S. W., \& Johnstone, R. M. 2005, MNRAS, 359, 1481

Berrington, R. C., Lugger, P. M., \& Cohn, H. N. 2002, AJ, 123, 2261

Bourdin, H., \& Mazzotta, P. 2008, A\&A, 479, 307

Briel, U. G., Finoguenov, A., \& Henry, J. P. 2004, A\&A, 426, 1

Brunetti, G., Cassano, R., Dolag, K., \& Setti, G. 2009, A\&A, 507, 661

Buote, D. A. 2001, ApJ, 553, L15

Burgett, W. S., Vick, M. M., Davis, D. S., et al. 2004, MNRAS, 352, 605

Burns, J. O., Loken, C., Gomez, P., et al. 1997, in Galactic Cluster Cooling Flows, ed. N. Soker, ASP Conf. Ser., 115, 21

Cappellari, M., \& Copin, Y. 2003, MNRAS, 342, 345

Cavagnolo, K. W., Donahue, M., Voit, G. M., \& Sun, M. 2009, ApJS, 182, 12

Chen, Y., Ikebe, Y., \& Böhringer, H. 2003, A\&A, 407, 41
Clowe, D., Bradač, M., Gonzalez, A. H., et al. 2006, ApJ, 648, L109

Coziol, R., Andernach, H., Caretta, C. A., Alamo-Martínez, K. A., \& Tago, E. 2009, AJ, 137, 4795

De Grandi, S., \& Molendi, S. 2009, A\&A, 508, 565

De Grandi, S., Ettori, S., Longhetti, M., \& Molendi, S. 2004, A\&A, 419, 7

De Luca, A., \& Molendi, S. 2004, A\&A, 419, 837

Dickey, J. M., \& Lockman F. J. 1990, ARA\&A, 28, 215

Diehl, S., \& Statler, T. S. 2006, MNRAS, 368, 497

Donahue, M., Voit, G. M., O’Dea, C. P., Baum, S. A., \& Sparks, W. B. 2005, ApJ, 630, L13

Dunn, R. J. H., \& Fabian, A. C. 2008, MNRAS, 385, 757

Dunn, R. J. H., Fabian, A. C., \& Taylor, G. B. 2005, MNRAS, 364, 1343

Edge, A. C., Stewart, G. C., Fabian, A. C., \& Arnaud, K. A. 1990, MNRAS, 245, 559

Ettori, S., De Grandi, S., \& Molendi, S. 2002, A\&A, 391, 841

Fabricant, D., Beers, T. C., Geller, M. J., et al. 1986, ApJ, 308, 530

Ferrari, C., Govoni, F., Schindler, S., Bykov, A. M., \& Rephaeli, Y. 2008, Space Sci. Rev., 134, 93

Finoguenov, A., Henriksen, M. J., Miniati, F., Briel, U. G., \& Jones, C. 2006, ApJ, 643, 790

Fusco-Femiano, R., Cavaliere, A., \& Lapi, A. 2009, ApJ, 705, 1019

Giacintucci, S., Mazzotta, P., Brunetti, G., Venturi, T., \& Bardelli, S. 2006, Astron. Nachr., 327, 573

Girardi, M., \& Mezzetti, M. 2001, ApJ, 548, 79

Gómez, P. L., Loken, C., Roettiger, K., \& Burns, J. O. 2002, ApJ, 569, 122

Gudehus, D. H., \& Hegyi, D. J. 1991, AJ, 101, 18

Henry, J. P., Finoguenov, A., \& Briel, U. G. 2004, ApJ, 615, 181

Johnston-Hollitt, M., Sato, M., Gill, J. A., Fleenor, M. C., \& Brick, A.-M. 2008, MNRAS, 390, 289

Kim, K.-T. 1999, J. Kor. Astron. Soc., 32, 75

Leccardi, A., \& Molendi, S. 2008a, A\&A, 487, 461

Leccardi, A., \& Molendi, S. 2008b, A\&A, 486, 359

Leccardi, A., Molendi, S., \& Rossetti, M. 2010, A\&A, 510, A82 (Paper I)

Lin, Y.-T., \& Mohr, J. J. 2004, ApJ, 617, 879

Maccacaro, T., Gioia, I. M., Wolter, A., Zamorani, G., \& Stocke, J. T. 1988, ApJ, 326, 680

McCarthy, I. G., Balogh, M. L., Babul, A., Poole, G. B., \& Horner, D. J. 2004, ApJ, 613, 811

McCarthy, I. G., Babul, A., Bower, R. G., \& Balogh, M. L. 2008, MNRAS, 386, 1309

Molendi, S., \& Pizzolato, F. 2001, ApJ, 560, 194

Motl, P. M., Burns, J. O., Loken, C., Norman, M. L., \& Bryan, G. 2004, ApJ, 606,635

O'Hara, T. B., Mohr, J. J., Bialek, J. J., \& Evrard, A. E. 2006, ApJ, 639, 64

Okabe, N., \& Umetsu, K. 2008, PASJ, 60, 345

Owers, M. S., Couch, W. J., \& Nulsen, P. E. J. 2009, ApJ, 693, 901

Peres, C. B., Fabian, A. C., Edge, A. C., et al. 1998, MNRAS, 298, 416

Peterson, J. R., \& Fabian, A. C. 2006, Phys. Rep., 427, 1

Peterson, J. R., Paerels, F. B. S., Kaastra, J. S., et al. 2001, A\&A, 365, L104

Ponman, T. J., Cannon, D. B., \& Navarro, J. F. 1999, Nature, 397, 135

Poole, G. B., Babul, A., McCarthy, I. G., Sanderson, A. J. R., \& Fardal, M. A. 2008, MNRAS, 391, 1163

Postman, M., \& Lauer, T. R. 1995, ApJ, 440, 28

Quintana, H., Ramirez, A., \& Way, M. J. 1996, AJ, 112, 36

Reiprich, T. H., Sarazin, C. L., Kempner, J. C., \& Tittley, E. 2004, ApJ, 608, 179

Ritchie, B. W., \& Thomas, P. A. 2002, MNRAS, 329, 675

Rossetti, M. 2006, Ph.D. Thesis, Univ. of Milano

Rossetti, M., Ghizzardi, S., Molendi, S., \& Finoguenov, A. 2007, A\&A, 463, 839

Rottgering, H. J. A., Wieringa, M. H., Hunstead, R. W., \& Ekers, R. D. 1997, MNRAS, 290, 577

Sanderson, A. J. R., Finoguenov, A., \& Mohr, J. J. 2005, ApJ, 630, 191

Sanderson, A. J. R., Ponman, T. J., \& O'Sullivan, E. 2006, MNRAS, 372, 1496

Sanderson, A. J. R., Edge, A. C., \& Smith, G. P. 2009a, MNRAS, 398, 1698

Sanderson, A. J. R., O'Sullivan, E., \& Ponman, T. J. 2009b, MNRAS, 428

Sarazin, C. L. 1988, X-ray emission from clusters of galaxies (Cambridge: Cambridge University Press), Cambridge Astrophys. Ser.

Sun, M., Murray, S. S., Markevitch, M., \& Vikhlinin, A. 2002, ApJ, 565, 867

Tamura, T., Bleeker, A., Kaastra, J., Ferrigno, C., \& Molendi, S. 2001, A\&A, 379,107

Venturi, T., Bardelli, S., Zagaria, M., Prandoni, I., \& Morganti, R. 2002, A\&A, 385,39

Venturi, T., Giacintucci, S., Dallacasa, D., et al. 2008, A\&A, 484, 327

Vikhlinin, A., Markevitch, M., \& Murray, S. S. 2001, ApJ, 551, 160

Vikhlinin, A., Burenin, R., Forman, W. R., et al. 2007, in Heating versus Cooling in Galaxies and Clusters of Galaxies, ed. H. Böhringer, G. W. Pratt, A. Finoguenov, \& P. Schuecker, 48

Voit, G. M., \& Donahue, M. 2005, ApJ, 634, 955 
Table 1. Observations analyzed for the clusters in the sample.

\begin{tabular}{|c|c|c|c|}
\hline Cluster & Observation ID & Exposure time $^{a}(\mathrm{ks})$ & Symbol $^{b}$ \\
\hline Abell 4038 & 0204460101 & 78.1 & Black open triangle \\
\hline \multirow[t]{3}{*}{ Abell 2199} & $0008030201^{c}$ & 42.8 & Black open square \\
\hline & 0008030301 & 11.2 & \\
\hline & 0008030601 & 11.3 & \\
\hline Abell 496 & 0135120201 & 47.2 & Black open circle \\
\hline $2 \mathrm{~A} 0335+096$ & 0147800201 & 230.8 & Black filled triangle \\
\hline Abell 2052 & 0109920101 & 85.1 & Black filled square \\
\hline \multirow[t]{2}{*}{ Abell 576} & $0205070401^{c}$ & 42.7 & Black filled circle \\
\hline & 0205070301 & 28.1 & \\
\hline Abell 3571 & 0086950201 & 43.5 & Blue open triangle \\
\hline Abell 119 & 0012440101 & 54.1 & Blue open square \\
\hline MKW3s & 0109930101 & 99.2 & Blue open circle \\
\hline Abell 1644 & 0010420201 & 42.0 & Blue filled triangle \\
\hline \multirow[t]{2}{*}{ Abell 4059} & $0109950101^{c}$ & 32.4 & Blue filled square \\
\hline & 0109950201 & 64.9 & \\
\hline Abell 3558 & 0107260101 & 126.4 & Blue filled circle \\
\hline \multirow[t]{5}{*}{ Abell 3562} & $0105261301^{c}$ & 116.6 & Red open triangle \\
\hline & 0105261501 & 45.0 & \\
\hline & 0105261601 & 52.5 & \\
\hline & 0105261701 & 57.7 & \\
\hline & 0105261801 & 23.6 & \\
\hline \multirow[t]{3}{*}{ Triangulum Australis } & $0093620101^{c}$ & 27.3 & Red open square \\
\hline & 0093620201 & 8.3 & \\
\hline & 0093620301 & 31.8 & \\
\hline Hydra A cluster & 0109980301 & 52.2 & Red open circle \\
\hline \multirow[t]{4}{*}{ Abell 754} & $0136740101^{c}$ & 40.6 & Light blue open square \\
\hline & 0136740201 & 14.8 & \\
\hline & 0112950401 & 31.8 & \\
\hline & 0112950301 & 34.8 & \\
\hline \multirow[t]{9}{*}{ Abell 3266} & $0105260901^{c}$ & 66.5 & Light blue open circle \\
\hline & 0105262001 & 13.7 & \\
\hline & 0105262101 & 16.2 & \\
\hline & 0105261101 & 33.1 & \\
\hline & 0105262201 & 9.1 & \\
\hline & 0105261001 & 5.8 & \\
\hline & 0105260701 & 56.4 & \\
\hline & 0105260801 & 56.7 & \\
\hline & 0105262501 & 17.3 & \\
\hline Abell 85 & 0065140101 & 34.6 & Red filled triangle \\
\hline Abell 3532 & 0030140301 & 27.7 & Green filled star \\
\hline \multirow[t]{7}{*}{ Abell 3667} & $0206850101^{c}$ & 162.9 & Light blue open triangle \\
\hline & 0105260101 & 15.4 & \\
\hline & 0105260601 & 62.5 & \\
\hline & 0105260401 & 45.6 & \\
\hline & 0105260301 & 47.2 & \\
\hline & 0105260501 & 38.6 & \\
\hline & 0105260201 & 31.8 & \\
\hline \multirow[t]{2}{*}{ Abell 2319} & $0302150101^{d}$ & $27.6^{e}$ & Red filled square \\
\hline & $0302150201^{d}$ & $28.2^{e}$ & \\
\hline \multirow[t]{5}{*}{ Abell 2256} & $0141380201^{c}$ & 33.1 & Light blue filled circle \\
\hline & 0112950601 & 25.3 & \\
\hline & 0112951501 & 28.1 & \\
\hline & 0112951601 & 30.9 & \\
\hline & 0141380101 & 24.4 & \\
\hline \multirow[t]{2}{*}{ Abell 3158} & $0300210201^{c}$ & $33.2^{e}$ & Red filled circle \\
\hline & 0300211301 & $14.0^{e}$ & \\
\hline Abell 1795 & 0097820101 & 97.3 & Green open triangle \\
\hline Abell 399 & 0112260101 & 27.9 & Green open square \\
\hline Abell 401 & 0112260301 & 34.7 & Green filled triangle \\
\hline Abell 3112 & 0105660101 & 64.6 & Green filled square \\
\hline Abell 2029 & 0111270201 & 30.8 & Green filled circle \\
\hline Abell 2255 & 0112260801 & 25.1 & Pink open triangle \\
\hline
\end{tabular}

Table 1. continued.

\begin{tabular}{cccc}
\hline \hline Cluster & Observation ID & Exposure time $^{a}(\mathrm{ks})$ & Symbol $^{b}$ \\
\hline Abell 1650 & 0093200101 & 75.0 & Pink open square \\
Abell 2597 & 0147330101 & 144.3 & Pink open circle \\
Abell 478 & 0109880101 & 136.7 & Pink filled triangle \\
PKS 0745-191 & 0105870101 & 34.3 & Pink filled square \\
Abell 2204 & $0112230301^{c}$ & 51.2 & Pink filled circle \\
& 0306490101 & $17.6^{e}$ & \\
& 0306490201 & $30.9^{e}$ & \\
& 0306490301 & $22.7^{e}$ & \\
Abell 1689 & 0306490401 & $29.7^{e}$ & \\
\hline
\end{tabular}

Notes. ${ }^{(a)}$ Sum of the exposure times of the three instruments after soft protons cleaning; ${ }^{(b)}$ symbols and colors used to mark each cluster in the figures of the present paper; ${ }^{(c)}$ observation used for spectral analysis in case of multiple pointings of the same object; ${ }^{(d)}$ we used both observations of A2319 to increase the statistics; ${ }^{(e)}$ since these observations have been performed after the degradation of CCD 6 in MOS1 in 2005, we did not consider the MOS1 detector and the exposure time given in the table is just MOS2 + pn.

Pages 13 to 25 are available in the electronic edition of the journal at http://wwW . aanda.org 


\section{Appendix A: Relation between pseudo-entropy and entropy ratios}

We have used the entropy profiles for the 31 clusters in our sample for which we have performed radial analysis to test if the pseudo-entropy ratios (defined in Paper I) are good indicators of the behavior of the true three dimensional entropy $\left(S \equiv T_{\mathrm{X}} / n_{\mathrm{e}}^{2 / 3}\right)$. We have therefore calculated the entropy ratio $S_{\text {IN }} / S_{\text {OUT }}$, starting from the three-dimensional entropy profiles obtained with the de-projected temperature and density profiles. We show in Fig. A.1 that there is a strong correlation between the "true" entropy ratio and the "pseudo" entropy ratios. We fitted the points in Fig. A.1 with a linear model taking into account the errors on both coordinates (with the IDL Astrolib procedure FITEXY.PRO ${ }^{5}$ ), and we found

$\frac{S_{\text {IN }}}{S_{\text {OUT }}}=-0.051 \pm 0.004+(0.792 \pm 0.011) * \frac{s_{\mathrm{IN}}}{s_{\mathrm{OUT}}}$.

As expected, the entropy ratios are smaller than pseudo-entropy ratios, since projection effects smooth out the gradients.

In order to quantify the scatter around the best fit relation, we have applied to the residuals a maximum likelihood algorithm that postulates a parent distribution described by a mean and an intrinsic dispersion (Maccacaro et al. 1988). To do this we have calculated an "effective error" on the $y$ coordinate, which takes into account the errors on both coordinates, defined as: $\sigma_{\text {eff }}^{2}=\sigma_{y}^{2}+b^{2} \sigma_{x}^{2}$, where $b$ is the best-fit value for the slope of the linear relation between the two data sets (in this case $b=0.792$, Eq. (A.1)). The mean value of the residual distribution is consistent with zero (0.021), with an intrinsic dispersion $\sigma=0.043$.

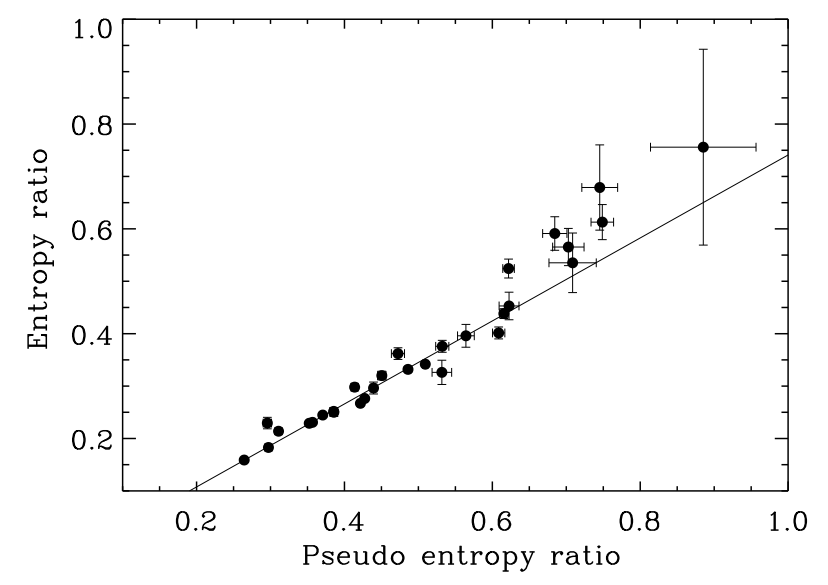

Fig. A.1. Entropy ratio versus pseudo-entropy ratio.

\section{Appendix B: Figures of individual clusters}

In this Appendix, we provide details for the low-entropy metal rich regions in the clusters with $\mathrm{CC}$ remnants. For each cluster, we show the EPIC X-ray flux image in the 0.4-2 keV energy range (see Rossetti et al. 2007, for details), the pseudo-entropy ratio image and the optical image taken from the DSS.

\footnotetext{
${ }^{5}$ http://idlastro.gsfc.nasa.gov/ftp/pro/math/fitexy . pro
} 


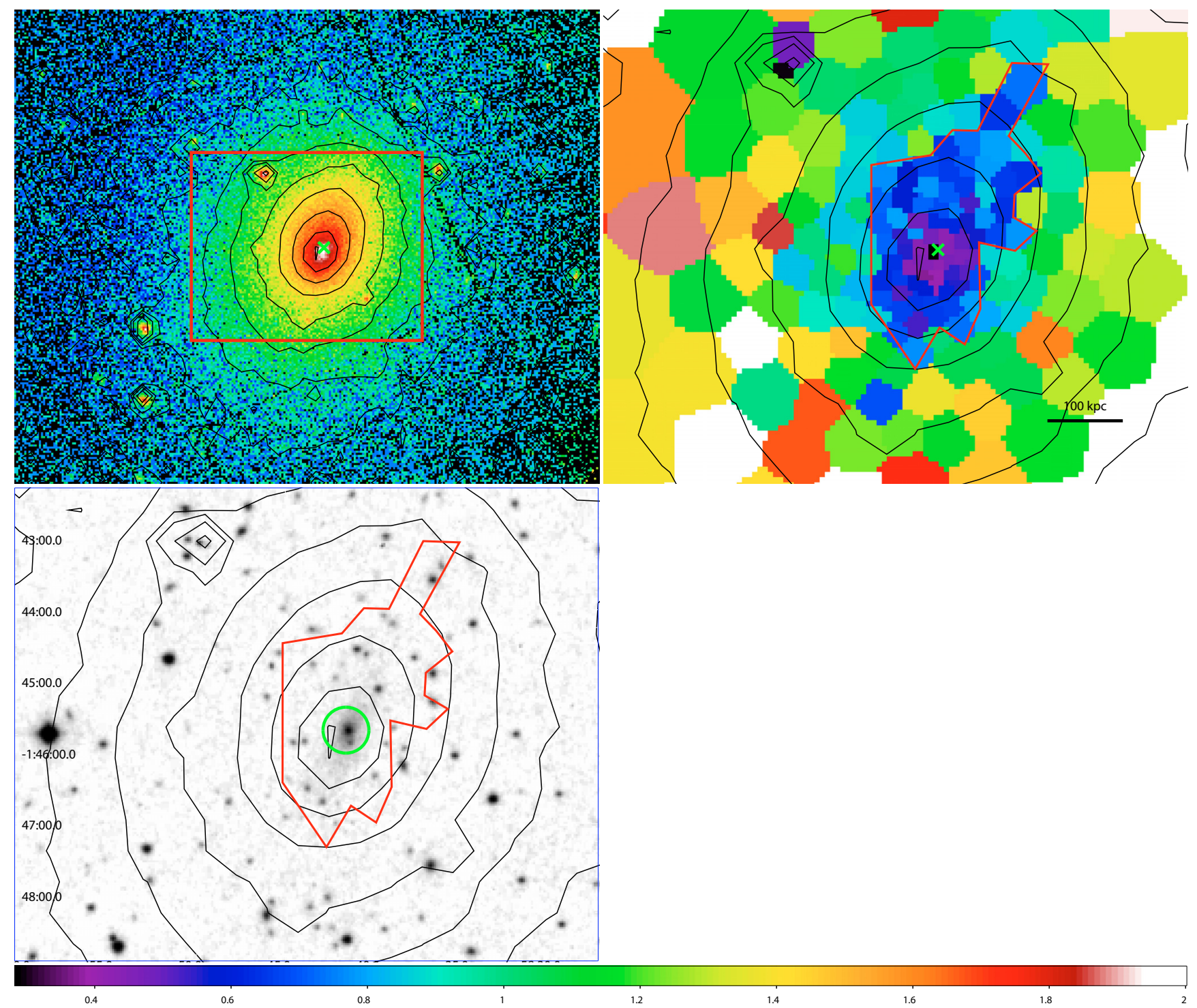

Fig. B.1. Abell 1650. Upper left panel: X-ray flux image. Upper right panel: pseudo-entropy ratio map (zoom), with X-ray contours overlaid. Lower left panel: optical image (zoom), with X-ray contours overlaid. The red box in the first panel marks the region zoomed in in the other two panels. The red polygon in the other two panels is the contour of the "CC-remnant", i.e. it marks the bins where the pseudo-entropy ratio is $<0.8$. The green circle (lower left) and " $\mathrm{X}$ " mark the position of the BCG. The scale refers to the pseudo-entropy ratio map. 


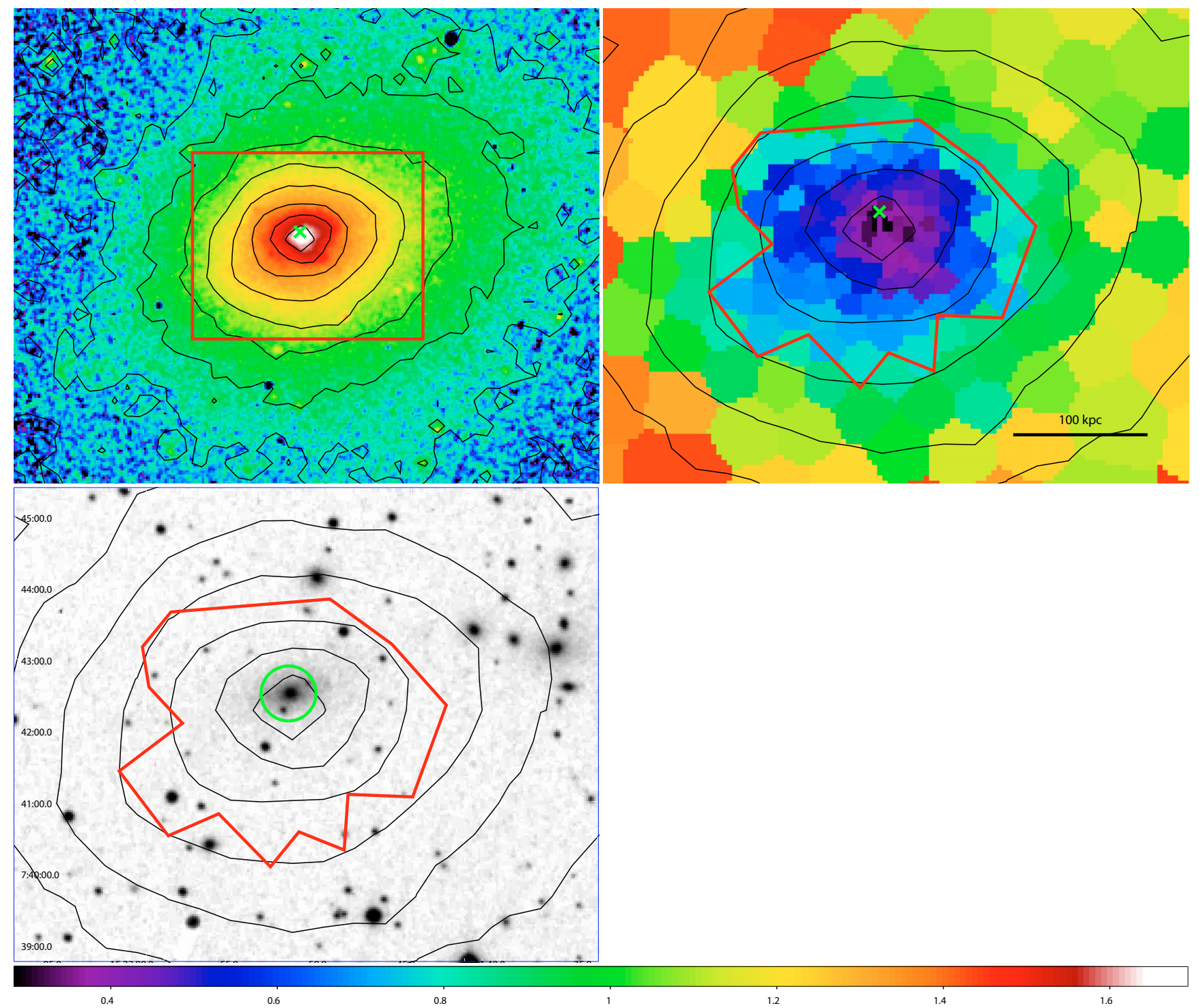

Fig. B.2. MKW3s. Upper left panel: X-ray flux image. Upper right panel: pseudo-entropy ratio map (zoom), with X-ray contours overlaid. Lower left panel: optical image (zoom), with X-ray contours overlaid. The red box in the first panel marks the region zoomed in in the other two panels. The red polygon in the other two panels is the contour of the "CC-remnant", i.e. it marks the bins where the pseudo-entropy ratio is $<0.8$. The green circle (lower left) and " $\mathrm{X}$ " mark the position of the BCG. The scale refers to the pseudo-entropy ratio map. 


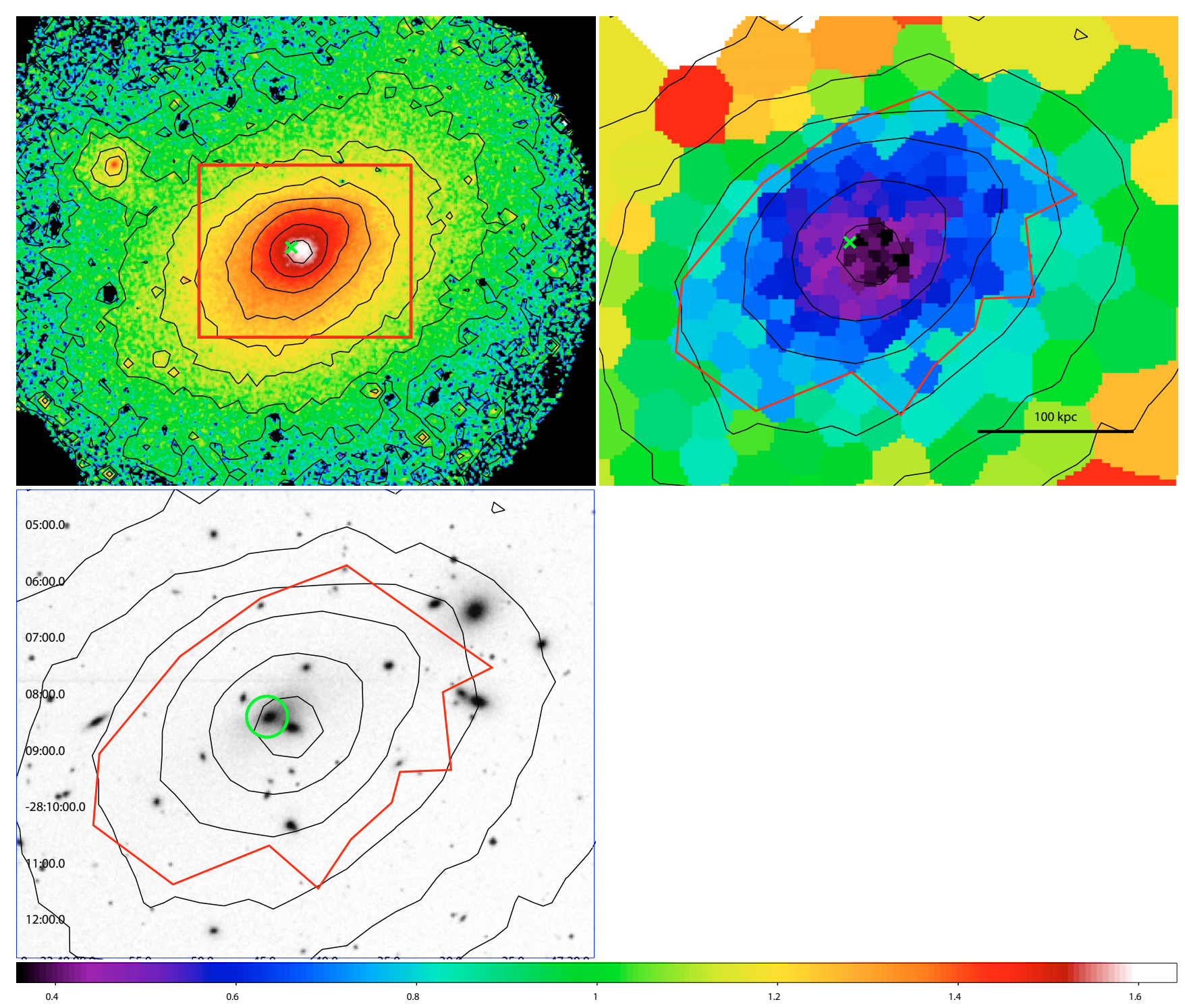

Fig. B.3. A4038. Upper left panel: X-ray flux image. Upper right panel: pseudo-entropy ratio map (zoom), with X-ray contours overlaid. Lower left panel: optical image (zoom), with X-ray contours overlaid. The red box in the first panel marks the region zoomed in in the other two panels. The red polygon in the other two panels is the contour of the "CC-remnant", i.e. it marks the bins where the pseudo-entropy ratio is $<0.8$. The green circle (lower left) and "X" mark the position of the BCG. The scale refers to the pseudo-entropy ratio map. 


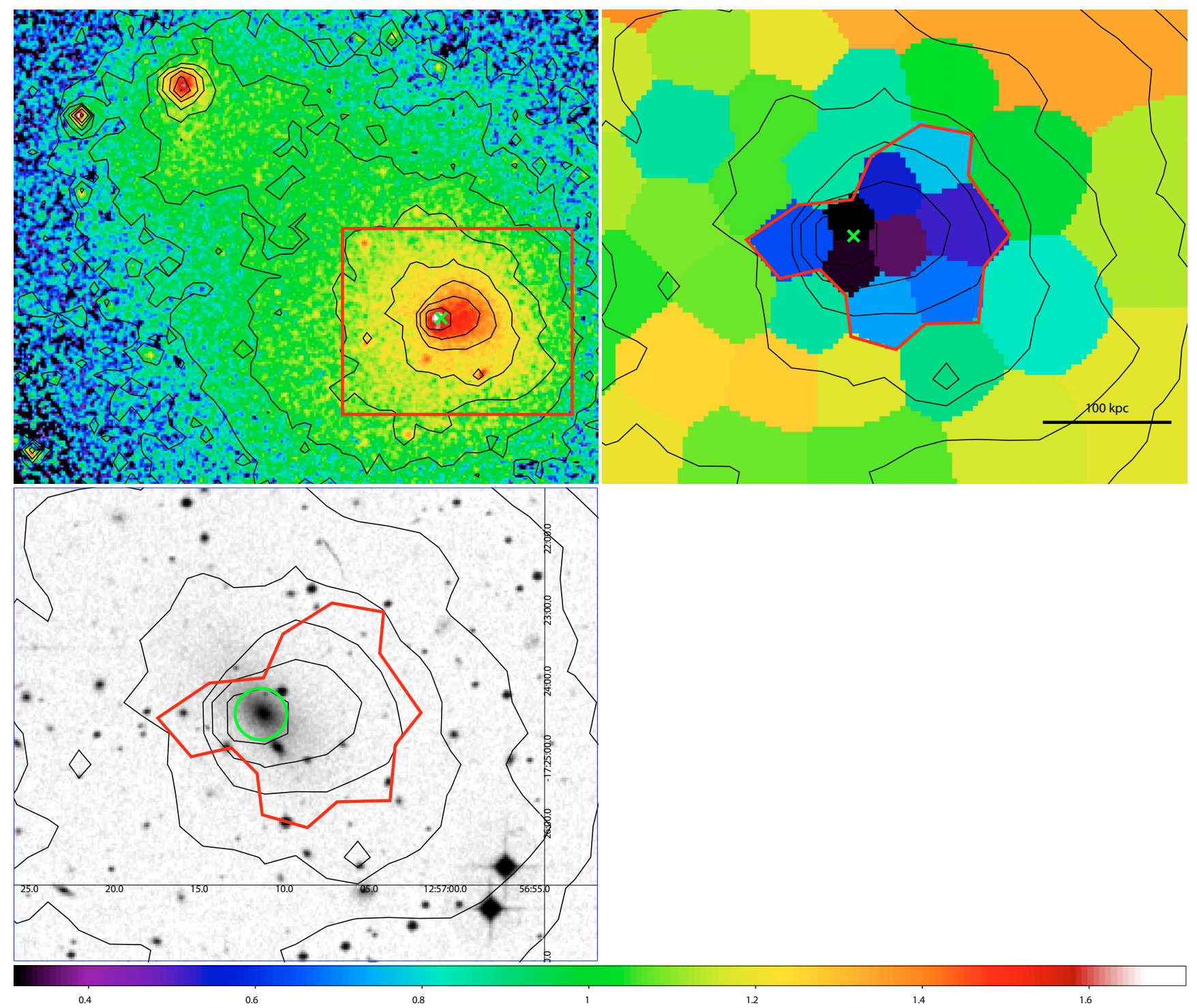

Fig. B.4. A1644. Upper left panel: X-ray flux image. Upper right panel: pseudo-entropy ratio map (zoom), with X-ray contours overlaid. Lower left panel: optical image (zoom), with X-ray contours overlaid. The red box in the first panel marks the region zoomed in in the other two panels. The red polygon in the other two panels is the contour of the "CC-remnant", i.e. it marks the bins where the pseudo-entropy ratio is $<0.8$. The green circle (lower left) and "X" mark the position of the BCG. The scale refers to the pseudo-entropy ratio map. 


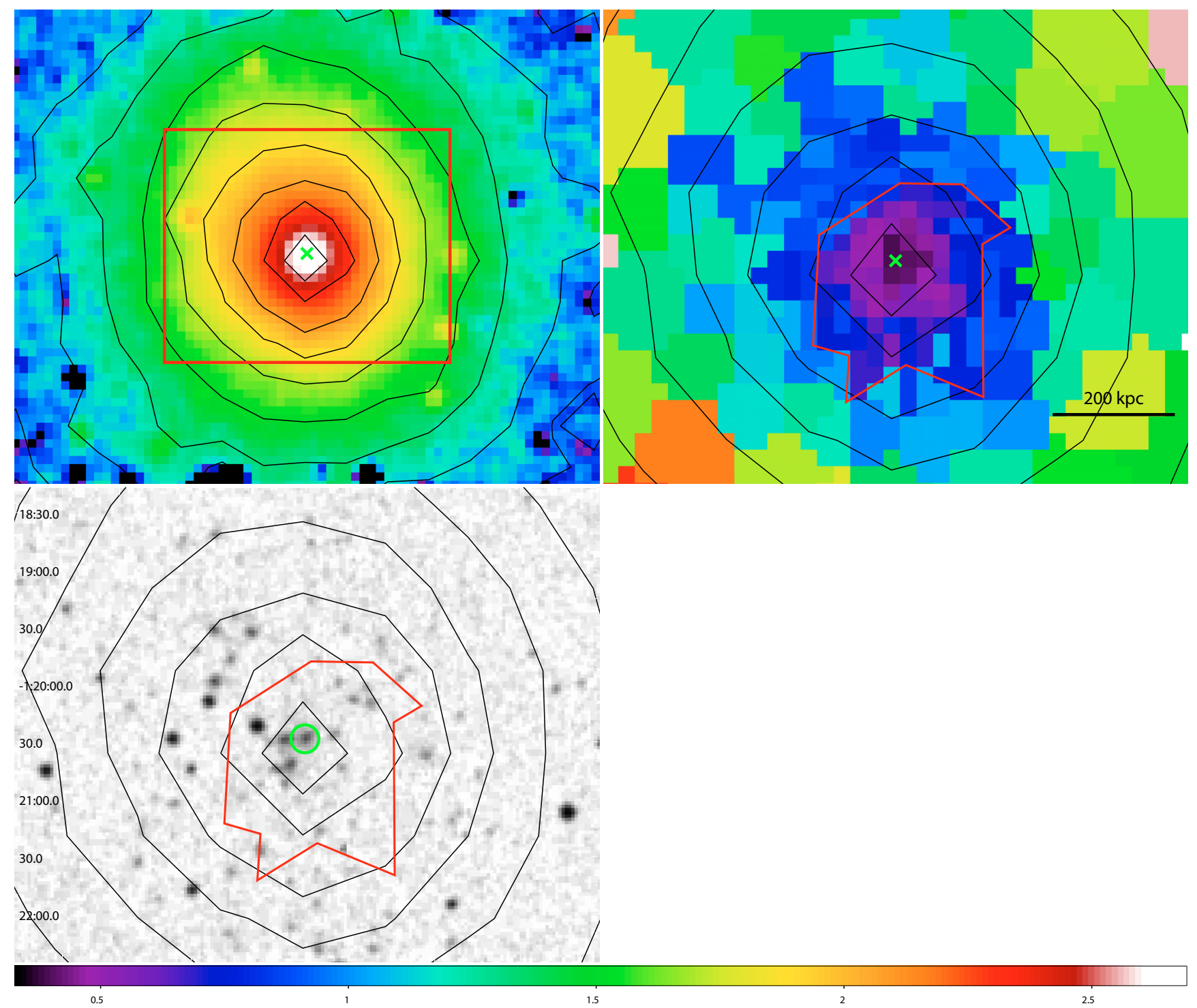

Fig. B.5. Abell 1689. Upper left panel: X-ray flux image. Upper right panel: pseudo-entropy ratio map (zoom), with X-ray contours overlaid. Lower left panel: optical image (zoom), with X-ray contours overlaid. The red box in the first panel marks the region zoomed in in the other two panels. The red polygon in the other two panels is the contour of the "CC-remnant", i.e. it marks the bins where the pseudo-entropy ratio is $<0.8$. The green circle (lower left) and " $\mathrm{X}$ " mark the position of the BCG. The scale refers to the pseudo-entropy ratio map. 


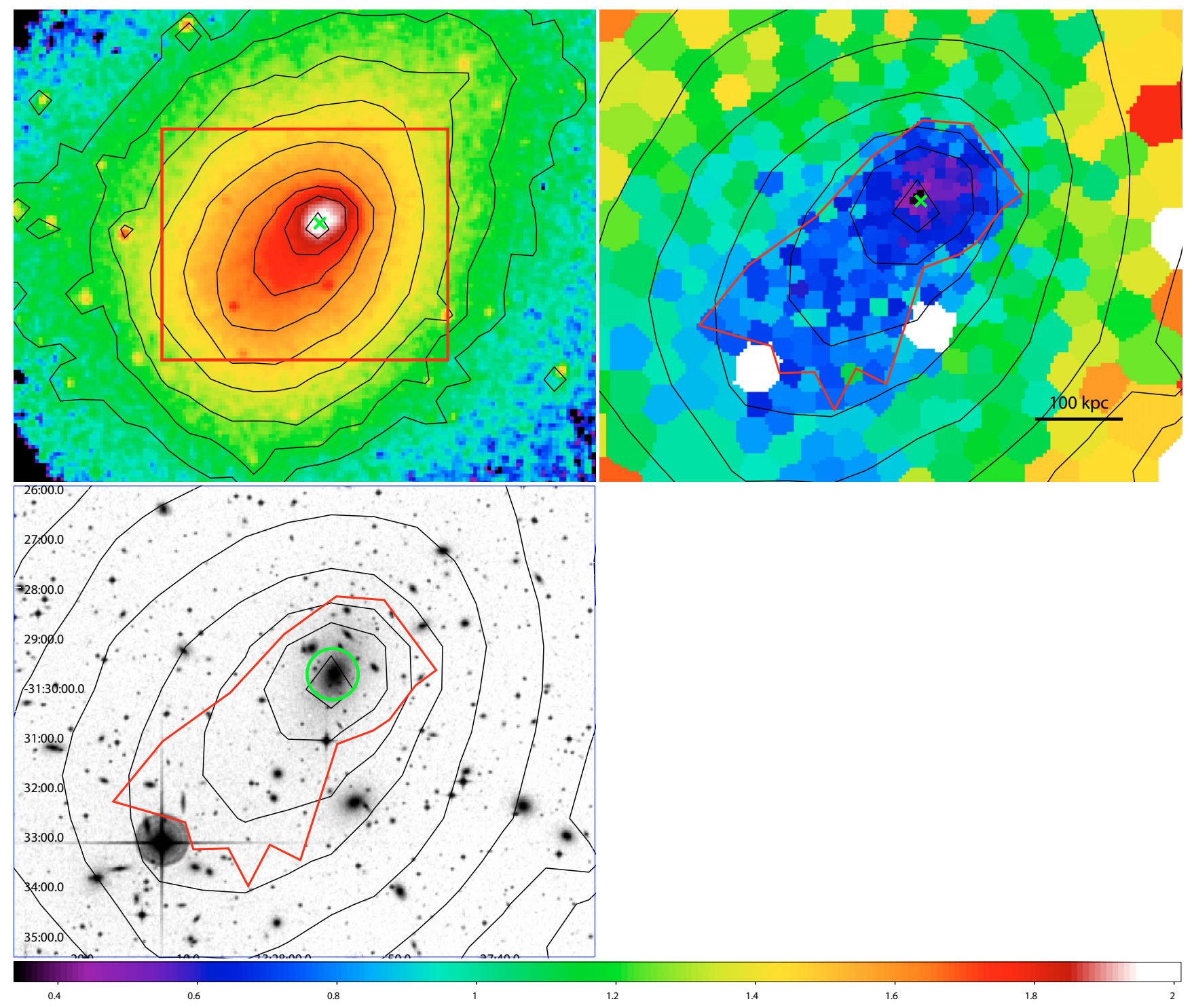

Fig. B.6. Abell 3558. Upper left panel: X-ray flux image. Upper right panel: pseudo-entropy ratio map (zoom), with X-ray contours overlaid. White regions have no data because of point sources subtraction (Rossetti et al. 2007). Lower left panel: optical image (zoom), with X-ray contours overlaid. The red box in the first panel marks the region zoomed in in the other two panels. The red polygon in the other two panels is the contour of the "CC-remnant", i.e. it marks the bins where the pseudo-entropy ratio is $<0.8$. The green circle (lower left) and "X" mark the position of the BCG. The scale refers to the pseudo-entropy ratio map. 


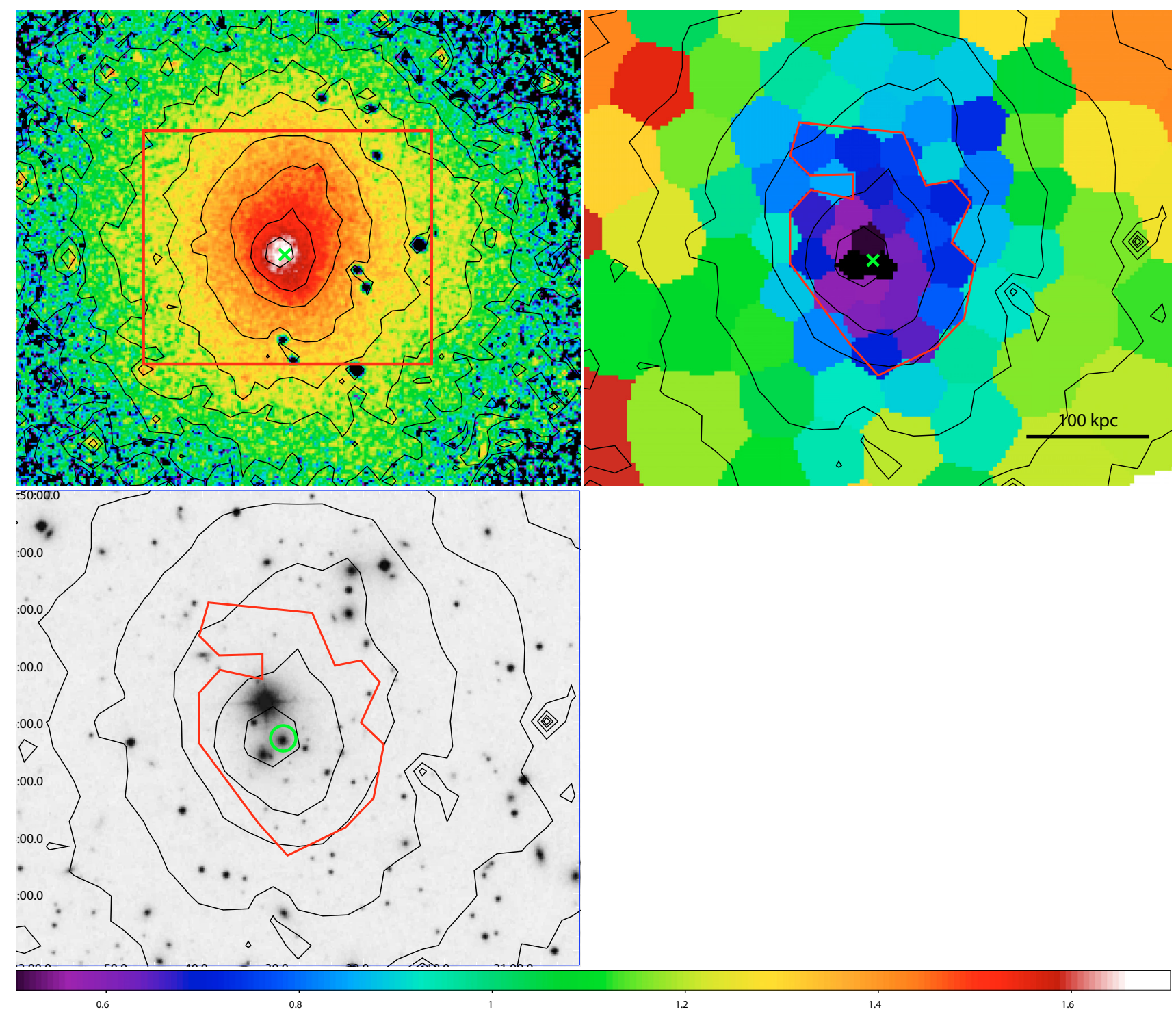

Fig. B.7. Abell 576. Upper left panel: X-ray flux image. Upper right panel: pseudo-entropy ratio map (zoom), with X-ray contours overlaid. Lower left panel: optical image (zoom), with X-ray contours overlaid. The red box in the first panel marks the region zoomed in in the other two panels. The red polygon in the other two panels is the contour of the "CC-remnant", i.e. it marks the bins where the pseudo-entropy ratio is $<0.8$. The green circle (lower left) and " $\mathrm{X}$ " mark the position of the BCG. The scale refers to the pseudo-entropy ratio map. 

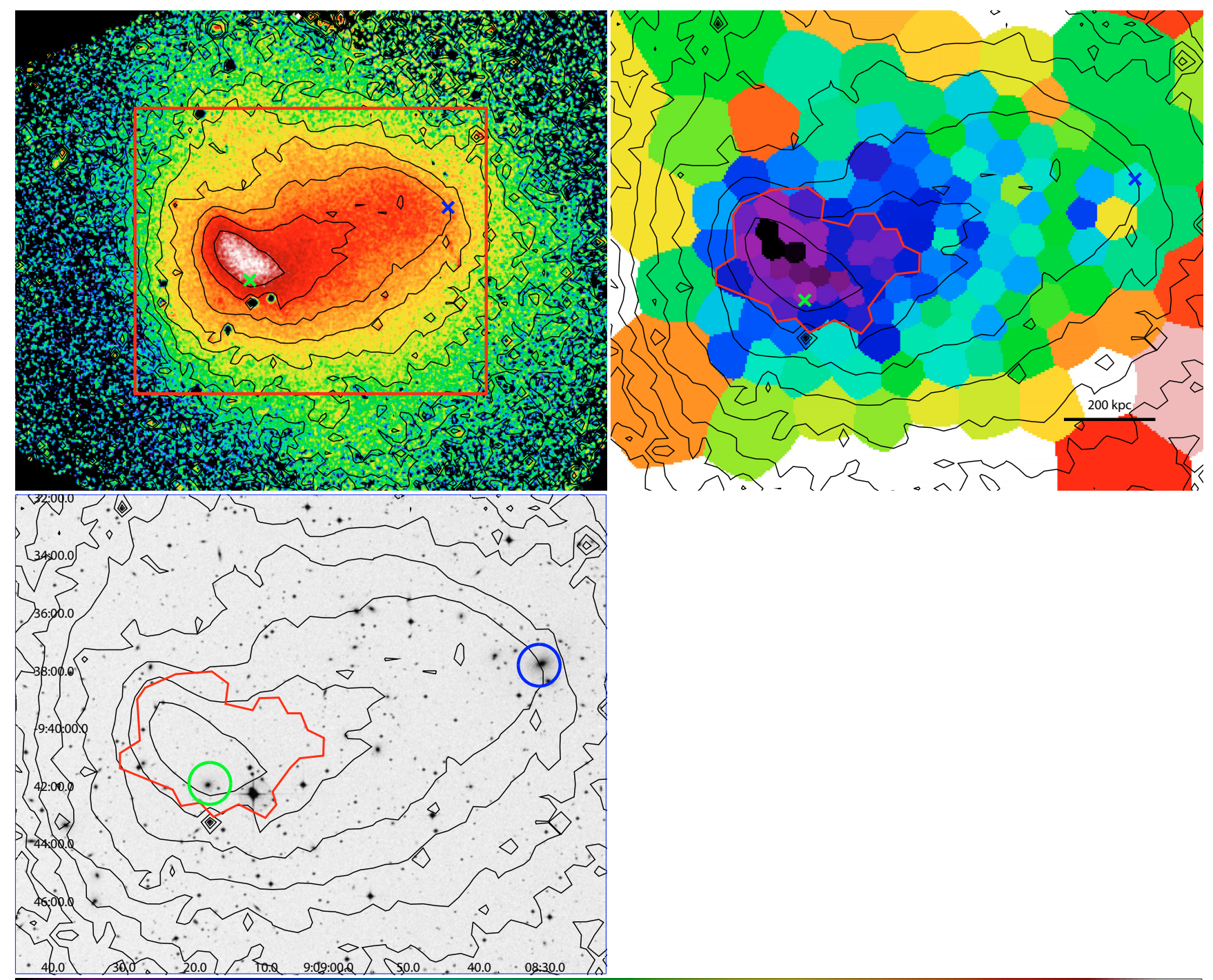

0.6

0.8

1.2

1.4

1.8

2.2

Fig. B.8. Abell 754. Upper left panel: X-ray flux image. Upper right panel: pseudo-entropy ratio map (zoom), with X-ray contours overlaid. Lower left panel: optical image (zoom), with X-ray contours overlaid. The red box in the first panel marks the region zoomed in in the other two panels. The red polygon in the upper right panel is the contour of the "CC-remnant", i.e. it marks the bins where the pseudo-entropy ratio is $<0.8$. The blue circle and X show the position of the BCG, while the green circle and X show the elliptical galaxy 2MASSX J09091923-0941591. The scale refers to the pseudo-entropy ratio map. 


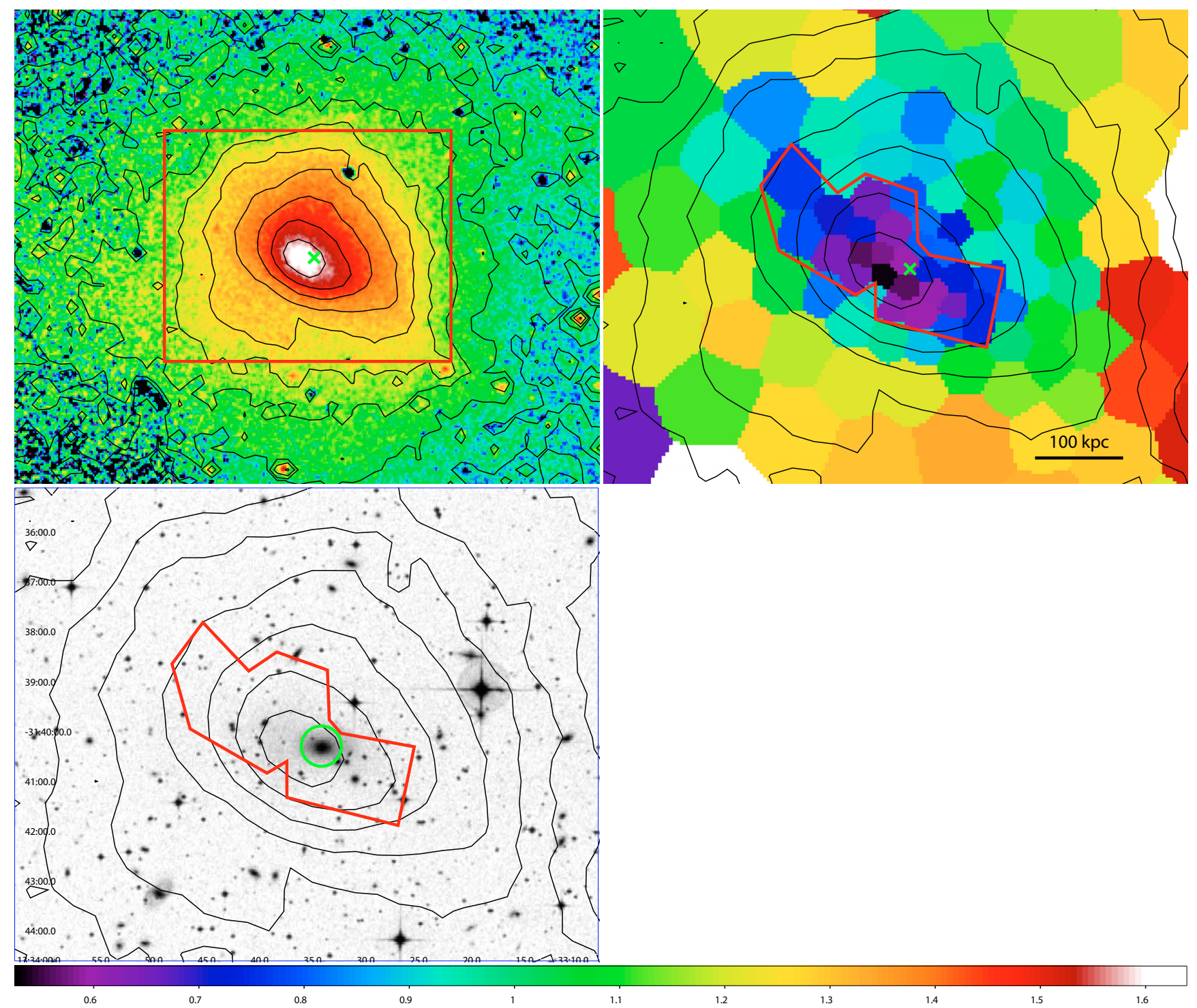

Fig. B.9. Abell 3562. Upper left panel: X-ray flux image. Upper right panel: pseudo-entropy ratio map (zoom), with X-ray contours overlaid. Lower left panel: optical image (zoom), with X-ray contours overlaid. The red box in the first panel marks the region zoomed in in the other two panels. The red polygon in the other two panels is the contour of the "CC-remnant", i.e. it marks the bins where the pseudo-entropy ratio is $<0.8$. The green circle (lower left) and " $\mathrm{X}$ " mark the position of the BCG. The scale refers to the pseudo-entropy ratio map. 


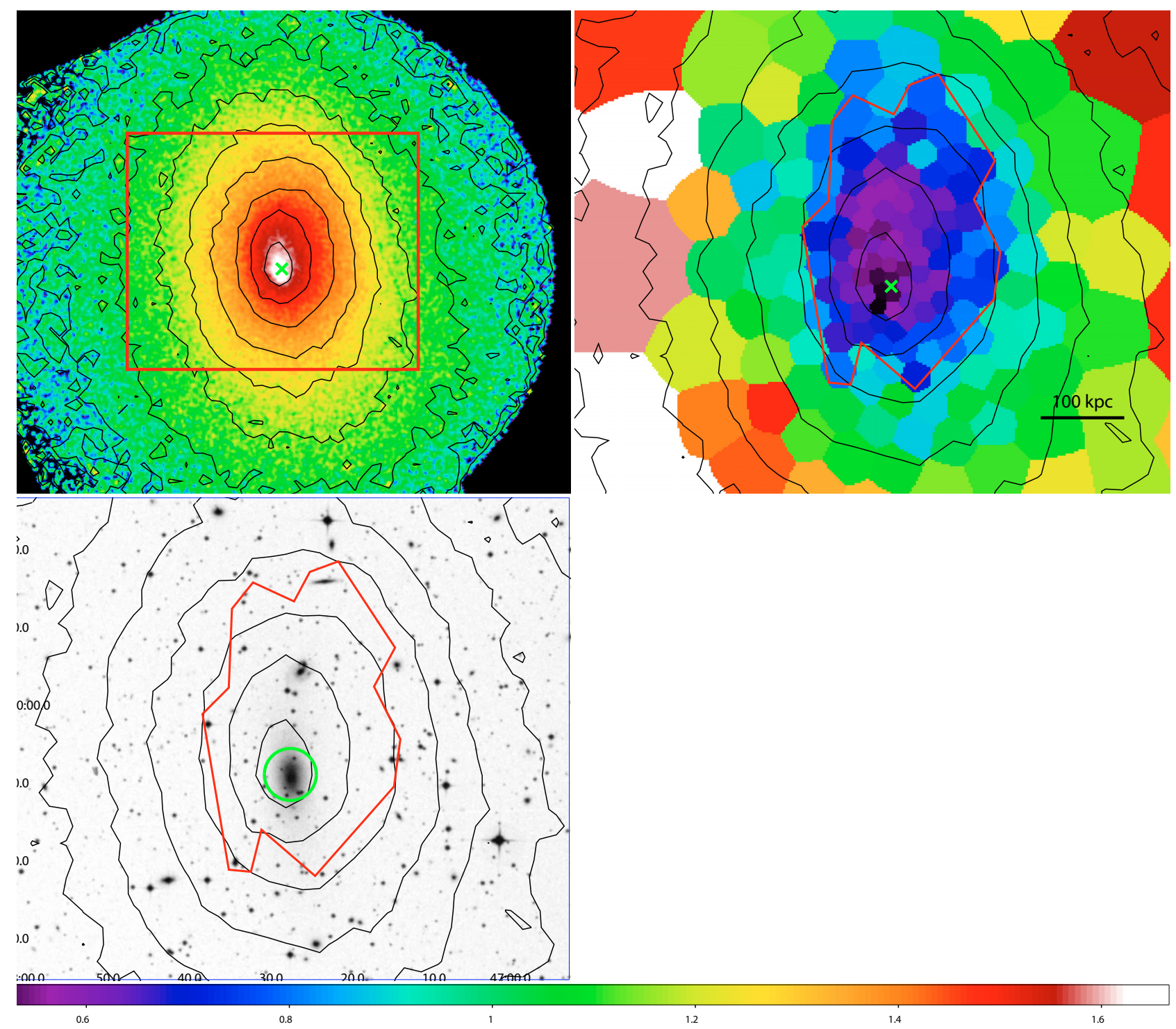

Fig. B.10. Abell 3571. Upper left panel: X-ray flux image. Upper right panel: pseudo-entropy ratio map (zoom), with X-ray contours overlaid. Lower left panel: optical image (zoom), with X-ray contours overlaid. The red box in the first panel marks the region zoomed in in the other two panels. The red polygon in the other two panels is the contour of the "CC-remnant", i.e. it marks the bins where the pseudo-entropy ratio is $<0.8$. The green circle (lower left) and " $\mathrm{X}$ " mark the position of the BCG. The scale refers to the pseudo-entropy ratio map. 


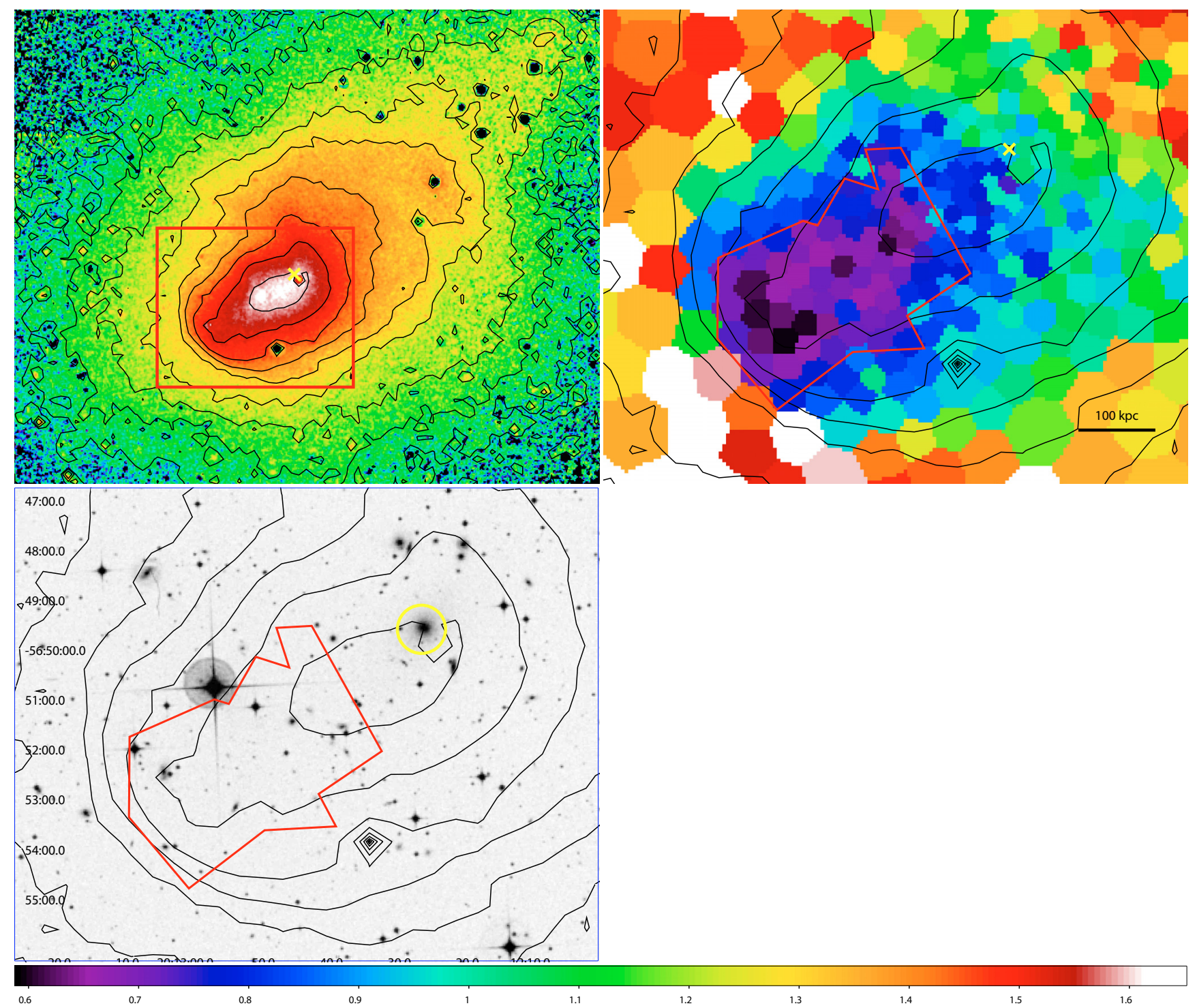

Fig. B.11. Abell 3667. Upper left panel: X-ray flux image. Upper right panel: pseudo entropy ratio map (zoom), with X-ray contours overlaid. Lower left panel: optical image (zoom), with X-ray contours overlaid. The red box in the first panel marks the region zoomed in in the other two panels. The red polygon in the other two panels is the contour of the "CC-remnant", i.e. it marks the bins where the pseudo entropy ratio is $<0.8$. The yellow circle (lower left) and "X" mark the position of the BCG. The scale refers to the pseudo entropy ratio map. 


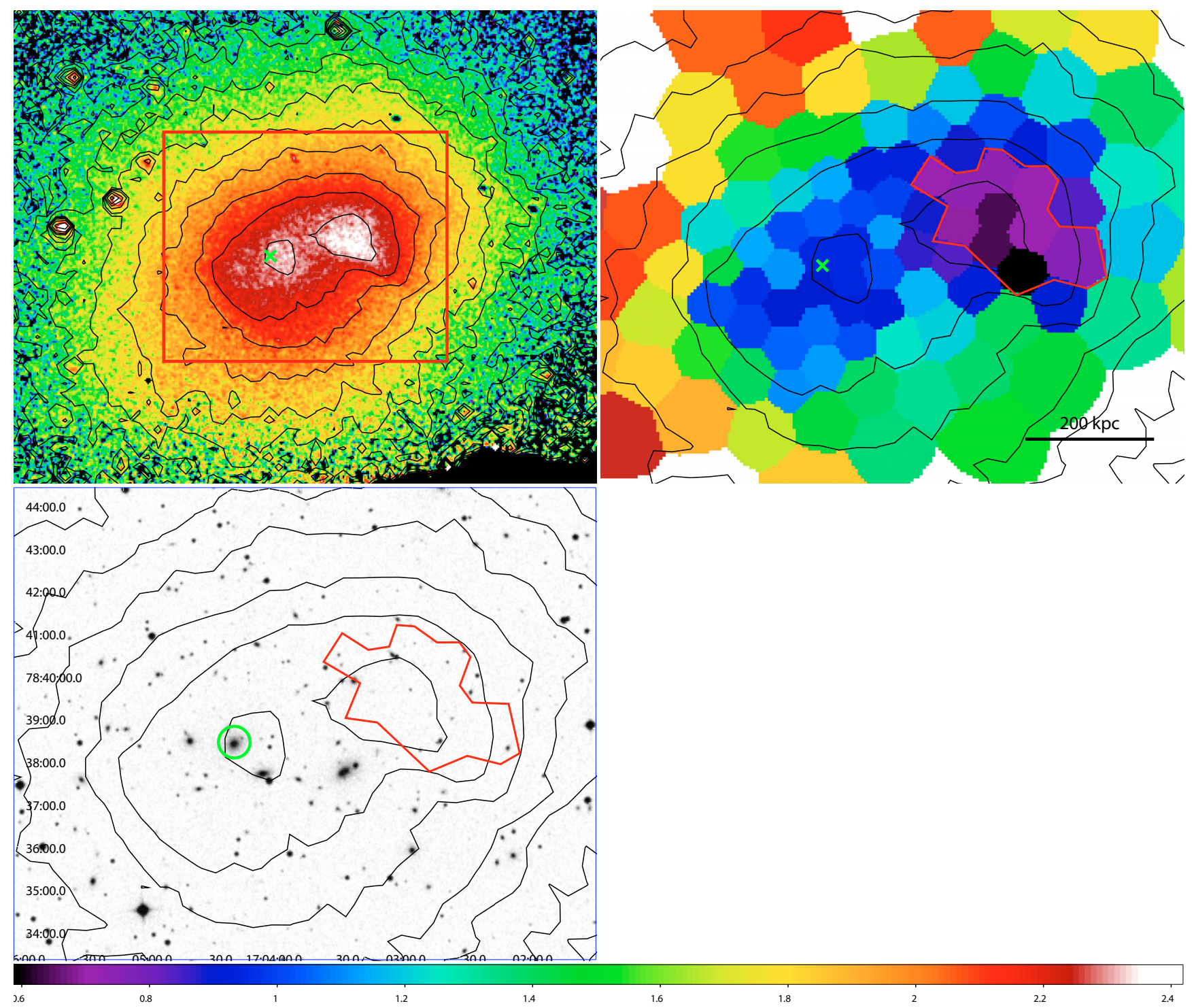

Fig. B.12. Abell 2256. Upper left panel: X-ray flux image. Upper right panel: pseudo-entropy ratio map (zoom), with X-ray contours overlaid. Lower left panel: optical image (zoom), with X-ray contours overlaid. The red box in the first panel marks the region zoomed in in the other two panels. The red polygon in the other two panels is the contour of the "CC-remnant", i.e. it marks the bins where the pseudo-entropy ratio is $<0.8$. The green circle (lower left) and "X" mark the position of the BCG. The scale refers to the pseudo-entropy ratio map. 